\title{
ACtividad CATALÍTICA DE COMPLEJOS $p$-R-CINAMATO DE Ce(III), Nd(III) Y Sm(III) EN LA OXIDACIÓN DE $\beta$-PINENO Y CANFENO
}

\author{
Catalytic activity of p-R-ethyl cinnamate Ce(III), Nd(III) And Sm(III) \\ COMPLEX IN THE OXIDATION OF $\beta$-PINENE AND CAMPHENE
}

Julianna Mazuera-Méndez ${ }^{*}$

Alberto Aragón-Muriel**

Dorian Polo-Cerón ${ }^{* * *}$

Recibido: 13 de agosto de 2017

Aceptado: 12 de septiembre de 2017

\section{Resumen}

Se realizó la síntesis de nueve complejos lantánidos de cerio, neodimio y samario con ligandos trans-cinamato (L1), trans- $p$-clorocinamato (L2) y trans- $p$-metoxicinamato (L3). La caracterización se realizó mediante espectroscopia FT-IR, análisis térmicos (TGA y DSC) y análisis elemental. Los compuestos obtenidos se utilizaron como catalizadores para la oxidación de canfeno y $\beta$-pineno, usando peróxido de hidrógeno (30\%) y ácido peracético (12\%) como agentes oxidantes. En la oxidación de canfeno, el complejo $\left[\mathrm{Nd}(L 2)_{3}\right]$ presentó la conversión más alta $(97.99 \%)$ con una selectividad del $84.05 \%$ hacia el ácido 3-canfenilocarboxílico, mientras que en la oxidación de $\beta$-pineno, el complejo $\left[\mathrm{Ce}(\mathrm{L} 3)_{3} \cdot 2 \mathrm{H}_{2} \mathrm{O}\right]$ presentó la conversión más alta $(82.62 \%)$ con una selectividad hacia la nopinona del $70.52 \%$.

Palabras clave: cinamato, lantánidos, actividad catalítica, canfeno, $\beta$-pineno.

\section{Abstract}

The synthesis of nine lanthanide complexes of cerium, neodymium and samarium with ligands trans-cinnamate (L1), trans-p-chlorocinnamate (L2) and trans-p-methoxycinnamate (L3) was carried out. The characterization performed by FT-IR spectroscopy, thermal analysis (TGA and DSC) and elemental analysis. The compounds obtained were used as catalysts for the oxidation of camphene and $\beta$-pinene, using hydrogen peroxide (30\%) and peracetic acid (12\%) as oxidizing agents. In the oxidation of camphene, the $\left[\mathrm{Nd}(L 2)_{3}\right]$ complex, showed the highest conversion (97.99\%) with a selectivity of $84.05 \%$ towards 3 -can phenyl carboxylic acid, while in $\beta$-pinene oxidation, the $\left[\mathrm{Ce}(L 3)_{3} 2 \mathrm{H}_{2} \mathrm{O}\right]$ complex, showed the highest conversion (82.62\%) with a selectivity towards the nopinone of $70.52 \%$.

Keywords: cinnamate, lanthanides, catalytic activity, camphene, $\beta$-pinene.

\footnotetext{
* Química. Departamento de Química, Universidad del Valle, Cali, Colombia. ORCID:http://orcid.org/0000-0001-89316383. julianna.mazuera@correounivalle.edu.co

** M. Sc. en Química. Departamento de Química, Universidad del Valle, Cali, Colombia. ORCID:http://orcid.org/00000001-5554-9592. alberto.aragon@correounivalle.edu.co

*** Ph. D. en Química. Departamento de Química, Universidad del Valle, Cali, Colombia. ORCID:http://orcid.org/00000002-6778-2209. dorian.polo@correounivalle.edu.co
} 


\section{INTRODUCCIÓN}

La oxidación de alquenos es uno de los procesos más importantes en la química orgánica, debido a que los productos generados, tales como aldehídos, alcoholes, cetonas u óxidos, entre otros, son precursores para la obtención de productos de alto valor agregado para la industria farmacéutica, cosmética, química fina, alimenticia, entre otras (Nalone et ál., 2015; Corma, Renz y Susarte, 2009; Belanger, 1998). Sin embargo, el uso en los procesos de agentes oxidantes tóxicos y la baja selectividad en los productos, sumado a los elevados costos de producción, son los principales obstáculos para la obtención de nuevas moléculas (Blaz y Pielichowski, 2006). Debido a esto, se buscan y plantean nuevos métodos de oxidación para la conversión de alquenos como $\beta$-pineno y canfeno en derivados que tengan aplicaciones en la industria y la investigación.

La oxidación de monoterpenos como el $\beta$-pineno y el canfeno es considerada un método importante para la valoración de estas abundantes y renovables olefinas naturales. Sin embargo, su producción a través de reacciones de oxidación no catalíticas generalmente se llevan a cabo con rendimientos bajos y baja selectividad (da Silva, Vieira, Oliveira y Ribeiro, 2013). Silva, RoblesDutenhefner, Menini y Gusevskaya (2003) reportaron que la oxidación de $\beta$-pineno, utilizando un catalizador de cobalto en una solución de ácido acético y acetonitrilo, produce una selectividad combinada inesperadamente alta, de hasta el $85 \%$ para los productos obtenidos; no obstante, mencionan que no se obtienen epóxidos ni derivados de glicol. Por otro lado, de Oliveira, Lopes y da Silva (2009) obtuvieron para la epoxidación de canfeno con catalizadores de paladio una baja conversión con alta selectividad hacia los epóxidos deseados (conversión $32 \%$ y el $90 \%$ de selectividad después de $12 \mathrm{~h}$ ). La oxidación de estos monoterpenos se ha estudiado empleando diferentes catalizadores, que incluyen metales de transición, como hierro (Caovilla et ál., 2008), titanio (Casuscelli et ál., 2008) y los soportes más comunes de rutenio y osmio (Stolle, 2013); sin embargo, pocos trabajos sobre oxidación de alquenos incluyen metales del bloque $f$. Los complejos cinamato de $\mathrm{Y}(\mathrm{III}), \mathrm{La}(\mathrm{III}), \mathrm{Ce}(\mathrm{III}), \mathrm{Nd}(\mathrm{III}), \mathrm{Sm}(\mathrm{III})$ e $\mathrm{Yb}(\mathrm{III})$ han sido objeto de investigación reciente para descubrir sus propiedades catalíticas. Aragón-Muriel et ál. (2014) demostraron que los complejos cinamato son una alternativa como catalizadores para la conversión de estireno a diferentes productos de oxidación; los autores obtuvieron en todos los casos conversiones superiores a $50 \%$ después de $19 \mathrm{~h}$ de reacción, y en algunos casos conversiones superiores al $95 \%$ con una alta selectividad para dos productos principales: benzaldehído y 1-feniletano-1,2-diol. En estas condiciones experimentales, se observó que es importante considerar el efecto del metal en las reacciones de oxidación del estireno; aquí se evidenció que el complejo con Ce(III) presentó la actividad catalítica más alta (cercana al $100 \%$ ) con una selectividad relativamente alta (72\%) para la conversión de estireno a 1-feniletano-1.2-diol en comparación con los otros metales. Esta investigación ha motivado la utilización de estos compuestos como catalizadores para la oxidación de $\beta$-pineno y canfeno, modificando las condiciones de reacción para de esta forma obtener mejores resultados catalíticos.

\section{Metodología}

\section{Reactivos}

Para la síntesis de los diferentes complejos se emplearon los ácidos trans-cinámico, trans- $p$-clorocinámico y trans- $p$-metoxicinámico, y los nitratos hidratados de cerio, neodimio y samario. Para las reacciones de oxidación catalítica se usaron los reactivos (+)-canfeno y $\beta$-pineno, los solventes acetonitrilo y tolueno y los agentes oxidantes peróxido de hidrógeno y ácido peracético. 


\section{Instrumentación}

Para la espectroscopia infrarroja transformadas de Fourier (FT-IR) se empleó un espectrofotómetro Shimadzu IRAffinity-1, mediante la técnica de reflectancia total atenuada (ATR) tomando los espectros en estado sólido. La relación porcentual de carbono e hidrógeno presente en cada uno de los compuestos obtenidos se realizó usando el equipo Thermo Scientific Analizer Flash EA 1112 Series. Para determinar las propiedades térmicas de los complejos se utilizó un analizador térmico simultáneo Mettler Toledo TGA/DSC 1 STAR System a una velocidad constante de calentamiento de $10^{\circ} \mathrm{C} / \mathrm{min}$ entre 25 y $900^{\circ} \mathrm{C}$ y bajo atmósfera de nitrógeno.

Para el análisis e identificación de los productos de oxidación se utilizó un sistema de cromatografía de gases acoplado a un espectrómetro de masas (GC-MS) Shimadzu QP2010, en el que se empleó una columna capilar DB-5 ms (30.0 $\mathrm{m} \mathrm{x} 0.25 \mathrm{~mm} \times 0.25 \mu \mathrm{m})$ y la técnica de ionización por impacto electrónico a $70 \mathrm{eV}$.

\section{Síntesis y caracterización}

Se sintetizó una serie de complejos lantánidos (Ce(III), Nd(III) y Sm(III)), usando sales derivadas de ácido trans-cinámico, ácido trans-p-clorocinámico y ácido trans-p-metoxicinámico como ligandos.

Los compuestos obtenidos se caracterizaron mediante espectroscopia FT-IR, análisis elemental $(\% \mathrm{C}, \% \mathrm{H})$, porcentaje de lantánido $(\% \mathrm{Ln})$ en el complejo por complexometría con EDTA, análisis termogravimétrico (TGA) y calorimetría diferencial de barrido (DSC).

\section{Preparación de los ligandos}

La preparación de los ligandos cinamato de sodio ( $L 1)$, p-clorocinamato de sodio (L2) y p-metoxicinamato de sodio (L3) se realizó mediante la reacción de neutralización de los ácidos transcinámico, trans- $p$-clorocinámico y trans- $p$-metoxicinámico con $\mathrm{NaOH}$. Las reacciones se llevaron a cabo a temperatura ambiente, en etanol y agitación constante por aproximadamente 30 minutos. Los compuestos obtenidos se filtraron y se secaron a temperaturas entre 60 y $100^{\circ} \mathrm{C}$ por 12 horas.

\section{Síntesis de los complejos cinamato de $\mathrm{Ce}(I I I), \mathrm{Nd}(I I I)$ y $\mathrm{Sm}(\mathrm{III})$}

Para la obtención de los complejos se utilizó un procedimiento similar al descrito por AragónMuriel, Upegui, Muñoz, Robledo y Polo-Cerón (2016): se usaron los ligandos preparados previamente y los lantánidos $\mathrm{Ce}(\mathrm{III}), \mathrm{Nd}$ (III) y $\mathrm{Sm}$ (III) que se encuentran en forma de nitratos hexahidratados. Las reacciones se llevaron a cabo a temperaturas entre 60 y $70^{\circ} \mathrm{C}$, en un sistema de reflujo con agitación constante durante 3.5 horas, utilizando metanol como solvente. Los compuestos obtenidos se filtraron, se lavaron varias veces con metanol y se secaron a temperaturas entre 90 y $100^{\circ} \mathrm{C}$ (ver figura 1$)$. 


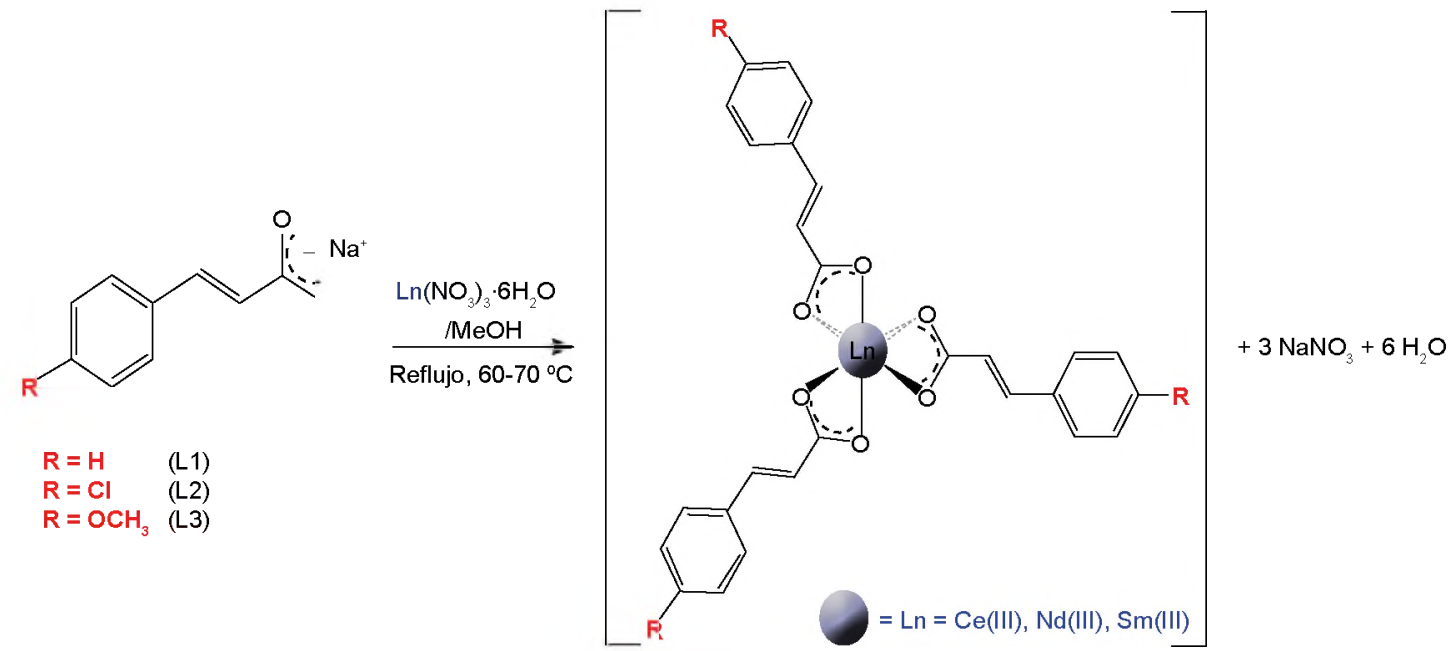

Figura 1. Reacción para la obtención de los complejos p-R-cinamato de Ce(III), Nd(III) y Sm(III).

\section{Sintesis de $\left.\left[\mathrm{Ce}(L 1)_{3} \cdot 0.5 \mathrm{H}_{2} \mathrm{O}\right](1)\right]$}

Para la síntesis del complejo, se utilizó el ligando L1 (877.6 mg; $5.158 \mathrm{mmol}$ ) previamente preparado, que se disolvió en $10 \mathrm{~mL}$ de metanol y a continuación se adicionó $\mathrm{Ce}\left(\mathrm{NO}_{3}\right)_{3} \cdot 6 \mathrm{H}_{2} \mathrm{O}(745.5 \mathrm{mg}$; $1.717 \mathrm{mmol})$. Al agregar el nitrato, la solución se tornó amarilla. Esta reacción se dejó en reflujo a $65^{\circ} \mathrm{C}$ con agitación durante 3.5 horas. Después de este tiempo, el compuesto obtenido se filtró y se secó a $90^{\circ} \mathrm{C}$. Finalmente, se obtuvo un sólido color amarillo. Rendimiento: $99.05 \%$ (960.1 mg). Fórmula molecular: $\mathrm{C}_{27} \mathrm{H}_{21} \mathrm{CeO}_{6}$ (masa molecular $=581.58 \mathrm{~g} / \mathrm{mol}$ ). Análisis elemental: C $55.06 \%\left(\mathrm{C}_{\text {teor }} 55.76 \%\right)$; H $3.57 \%$ (3.64\%); Ce 24.29\% (24.09\%). IR (ATR, $\left.\mathrm{cm}^{-1}\right): 3051.39 \mathrm{vw}$; $3008.95 \mathrm{vw} ; 1633.71 \mathrm{~s} ; 1490.97 \mathrm{vs} ; 1448.54 \mathrm{~s} ; 1384.89 \mathrm{~s} ; 1240.23 \mathrm{~s} ; 981.77 \mathrm{~s} ; 877.61 \mathrm{~s} ; 850.61 \mathrm{~s} ;$ $777.31 \mathrm{~s} ; 738.74 \mathrm{~s} ; 727.16 \mathrm{~s} ; 686.66 \mathrm{~s}$. TGA pérdida de masa: $1.59 \%$ (280-340 ${ }^{\circ} \mathrm{C}, 1$ paso, calc. $0.5 \times \mathrm{H}_{2} \mathrm{O}=1.53 \%$ ). Solubilidad en agua: $7.74 \times 10^{-4} \mathrm{~mol} \mathrm{~L}^{-1}$.

Los complejos (2-9) se sintetizaron de forma análoga al compuesto (1).

$\left[\mathrm{Nd}(\mathrm{L} 1)_{3} \cdot 0.4 \mathrm{H}_{2} \mathrm{O}\right](2)$

Sólido violeta claro. Rendimiento: $90.54 \%$ (904.7 mg). Fórmula molecular: $\mathrm{C}_{27} \mathrm{H}_{21} \mathrm{NdO}_{6}$ (masa molecular $=585.70 \mathrm{~g} / \mathrm{mol})$. Análisis elemental: C $54.53 \%\left(\mathrm{C}_{\text {teor }} 55.37 \%\right) ; \mathrm{H} 3.64 \%$ (3.61 \%); Nd 25.29 \% (24.63\%). IR (ATR, $\left.\mathrm{cm}^{-1}\right): 3053.32 \mathrm{vw} ; 3008.95 \mathrm{vw} ; 1633.71 \mathrm{~s} ; 1490.97 \mathrm{vs} ; 1446.61 \mathrm{~s} ; 1386.82 \mathrm{~s} ;$ $1240.23 \mathrm{~s} ; 981.77 \mathrm{~s} ; 877.61 \mathrm{~s} ; 850.61 \mathrm{~s} ; 779.24 \mathrm{~s} ; 740.67 \mathrm{~s} ; 729.09 \mathrm{~s} ; 686.66 \mathrm{~s}$. TGA pérdida de masa: $1.17 \%\left(280-330^{\circ} \mathrm{C}, 1\right.$ paso, calc. $\left.0.4 \mathrm{x} \mathrm{H}_{2} \mathrm{O}=1.22 \%\right)$. Solubilidad en agua: $7.26 \times 10^{-4} \mathrm{~mol} \mathrm{~L}^{-1}$.

$\left[\mathrm{Sm}(\mathrm{L} 1)_{3} \cdot 0.5 \mathrm{H}_{2} \mathrm{O}\right](3)$

Sólido blanco. Rendimiento 90.70 \% (907.2 mg). Fórmula molecular $\mathrm{C}_{27} \mathrm{H}_{21} \mathrm{SmO}_{6}$ (masa molecular $=591.82 \mathrm{~g} / \mathrm{mol})$. Análisis elemental: C $53.98 \%\left(\mathrm{C}_{\text {teor }} 54.80 \%\right) ; \mathrm{H} 3.57 \%(3.58 \%) ; \mathrm{Sm}$ $23.64 \%$ (25.41\%). IR (ATR, cm cm $^{-1} 3055.24 \mathrm{vw} ; 3008.95 \mathrm{vw} ; 1633.71 \mathrm{~s} ; 1490.97 \mathrm{vs;} 1448.54 \mathrm{~s}$; 1390.68 s; 1240.23 s; 981.77 s; 879.77 s; 850.61 s; 779.24 s; 740.67 s; 729.09 s; 686.66 s. TGA pérdida de masa: $1.30 \%\left(280-340{ }^{\circ} \mathrm{C}, 1\right.$ paso, calc. $\left.0.5 \times \mathrm{H}_{2} \mathrm{O}=1.50 \%\right)$. Solubilidad en agua: $2.62 \times 10^{-4} \mathrm{~mol} \mathrm{~L}^{-1}$. 
$\left[\mathrm{Ce}(\mathrm{L} 2)_{3} \cdot \mathrm{H}_{2} \mathrm{O}\right](4)$

Sólido amarillo. Rendimiento $92.6 \%$ (973.6 mg). Fórmula molecular $\mathrm{C}_{27} \mathrm{H}_{20} \mathrm{CeCl}_{3} \mathrm{O}_{7}$ (Masa molecular $=702.92 \mathrm{~g} / \mathrm{mol})$. Análisis elemental: C $45.00 \%\left(\mathrm{C}_{\text {teor }} 46.14 \%\right) ; \mathrm{H} 2.56 \%(2.87 \%) ; \mathrm{Ce}$ $18.57 \%$ (19.93 \%). IR (ATR, $\mathrm{cm}^{-1}$ ): $3369.64 \mathrm{w} ; 3030.17 \mathrm{vw} ; 1635.64 \mathrm{~s} ; 1593.20 \mathrm{w} ; 1500.62 \mathrm{~s}$; 1489.05 vs; 1409.96 vs; 1386.82 vs; $1280.73 \mathrm{w} ; 1246.02 \mathrm{~s} ; 1176.58 \mathrm{w} ; 1087.85$ vs; $1012.63 \mathrm{~s} ;$ $979.84 \mathrm{~s} ; 943.19 \mathrm{w} ; 879.54 \mathrm{w} ; 856.39 \mathrm{vw} ; 821.68 \mathrm{vs} ; 736.81 \mathrm{~s} ; 690.52 \mathrm{w} ; 667.37 \mathrm{w} ; 611.43 \mathrm{w}$. TGA pérdida de masa: $2.76 \%\left(280-330^{\circ} \mathrm{C}, 1\right.$ paso, calc. $\left.1 \mathrm{x}_{2} \mathrm{O}=2.56 \%\right)$. Solubilidad en agua: $2.35 \times 10^{-4} \mathrm{~mol} \mathrm{~L}^{-1}$.

\section{$\left[N d(L 2)_{3}\right](5)$}

Sólido violeta claro. Rendimiento $82.14 \%$ (843.4 mg). Fórmula molecular $\mathrm{C}_{27} \mathrm{H}_{18} \mathrm{NdCl}_{3} \mathrm{O}_{6}$ (masa molecular $=689.03 \mathrm{~g} / \mathrm{mol})$. Análisis elemental: $\mathrm{C} 45.69 \%\left(\mathrm{C}_{\text {teor }} 47.07 \%\right) ; \mathrm{H} 2.56 \%(2.63 \%) ; \mathrm{Nd}$ $20.31 \%$ (20.93\%). IR (ATR, cm $\left.{ }^{-1}\right): 3028.24 \mathrm{vw} ; 2360.87 \mathrm{vw} ; 1635.64 \mathrm{~s} ; 1489.05 \mathrm{vs;} 1409.96 \mathrm{~s}$; 1384.89 vs; 1244.09 s; 1087.85 vs; 1012.63 s; 977.91 s; 821.68 vs; 752.24 s; 736.81 s; 725.23 s; $690.52 \mathrm{w} ; 667.37 \mathrm{w}$. Solubilidad en agua: $1.89 \times 10^{-4} \mathrm{~mol} \mathrm{~L}^{-1}$.

\section{$\left[\mathrm{Sm}(\mathrm{L2})_{3}\right] \cdot 2 \mathrm{H}_{2} \mathrm{O}(6)$}

Sólido blanco. Rendimiento $91.02 \%$ (990.0 mg). Fórmula molecular $\mathrm{C}_{27} \mathrm{H}_{22} \mathrm{SmCl}_{3} \mathrm{O}_{8}$ (masa molecular $=731.17 \mathrm{~g} / \mathrm{mol})$. Análisis elemental: C 42.50\% ( $\left.\mathrm{C}_{\text {teor }} 44.35 \%\right) ; \mathrm{H} 2.60 \%$ (3.03 \%); Sm $22.86 \%$ (20.56 \%). IR (ATR, cm${ }^{-1}$ ): 3604.96 s; $3053.32 \mathrm{vw} ; 1635.64 \mathrm{vs;} 1591.27 \mathrm{w} ; 1560.41$ s; 1539.20 s; 1521.84 s; 1489.04 vs; 1379.10 vs; 1278.81 s; 1234.44 s; 1176.58 w; 1112.93 w; 1087.85 vs; $1012.63 \mathrm{~s} ; 991.41 \mathrm{w} ; 966.34 \mathrm{~s} ; 887.26 \mathrm{w} ; 862.18 \mathrm{w} ; 819.75 \mathrm{vs} ; 752.24 \mathrm{~s} ; 742.59 \mathrm{~s} ;$ $719.45 \mathrm{~s} ; 688.59 \mathrm{w} ; 665.44 \mathrm{~s} ; 632.65 \mathrm{vw}$. TGA pérdida de masa: $4.87 \%\left(100-280^{\circ} \mathrm{C}, 1\right.$ paso, calc. $\left.2 \times \mathrm{H}_{2} \mathrm{O}=4.92 \%\right)$. Solubilidad en agua: $6.43 \times 10^{-4} \mathrm{~mol} \mathrm{~L}^{-1}$.

$\left[\mathrm{Ce}(\mathrm{L} 3)_{3} \cdot \mathrm{H}_{2} \mathrm{O}\right] \cdot \mathrm{H}_{2} \mathrm{O}(7)$

Sólido amarillo. Rendimiento $88.60 \%$ (967.0 mg). Fórmula molecular $\mathrm{C}_{30} \mathrm{H}_{31} \mathrm{CeO}_{11}$ (masa molecular $=707.68 \mathrm{~g} / \mathrm{mol})$. Análisis elemental: C $49.93 \%\left(\mathrm{C}_{\text {teor }} 50.92 \%\right) ; \mathrm{H} 3.95 \%$ (4.42\%); Ce $21.44 \%$ (19.80\%). IR (ATR, cm $\left.{ }^{-1}\right): 3502.73 \mathrm{w} ; 3032.10 \mathrm{vw} ; 2931.80 \mathrm{vw} ; 2839.22 \mathrm{vw} ; 1680.00 \mathrm{~s} ; 1633.71$ s; 1604.77 s; 1571.99 s; 1508.33 vs; 1458.18 w; 1423.47 s; 1386.82 vs; 1305.81 s; 1292.31 s; 1240.23 vs; 1170.79 vs; 1109.07 w; 1026.13 s; 983.70 s; 829.39 vs; 779.24 s; 721.38 s; $696.30 \mathrm{w}$; 636.51 w. TGA pérdida de masa: $2.56 \%\left(30-190^{\circ} \mathrm{C}, 1\right.$ paso, calc. $\left.1 \times \mathrm{H}_{2} \mathrm{O}=2.54 \%\right), 2.30 \%$ (190$320^{\circ} \mathrm{C}, 2$ paso, calc. $1 \times \mathrm{H}_{2} \mathrm{O}=2.54 \%$ ). Solubilidad en agua: $5.30 \times 10^{-4} \mathrm{~mol} \mathrm{~L}^{-1}$.

\section{$\left[\mathrm{Nd}(\mathrm{L} 3)_{3}\right] \cdot \mathrm{H}_{2} \mathrm{O}(8)$}

Sólido violeta claro. Rendimiento $94.38 \%$ (1030.7 mg). Fórmula molecular $\mathrm{C}_{30} \mathrm{H}_{29} \mathrm{NdO}_{10}$ (masa molecular $=693.79 \mathrm{~g} / \mathrm{mol})$. Análisis elemental: C $48.57 \%\left(\mathrm{C}_{\text {teor }} 51.94 \%\right) ; \mathrm{H} 3.99 \%$ (4.21\%); Nd 23.33\% (20.79\%). IR (ATR, $\left.\mathrm{cm}^{-1}\right): 3562.52 \mathrm{w} ; 3456.44 \mathrm{w} ; 2958.80 \mathrm{vw} ; 2931.80$ vw; $1649.14 \mathrm{~s} ; 1633.71 \mathrm{~s} ; 1602.85 \mathrm{~s} ; 1562.34 \mathrm{~s} ; 1508.33 \mathrm{~s} ; 1458.18 \mathrm{w} ; 1425.40 \mathrm{~s} ; 1388.75 \mathrm{vs} ;$ 1300.02 s; 1242.16 vs; 1172.72 vs; $1111.00 \mathrm{w} ; 1026.13$ s; $985.62 \mathrm{~s} ; 883.40 \mathrm{w} ; 833.25$ vs; $779.24 \mathrm{~s}$; 723.31 s. TGA pérdida de masa: $2.63 \%\left(30-140{ }^{\circ} \mathrm{C}, 1\right.$ paso, calc. $\left.1 \mathrm{x}_{2} \mathrm{O}=2.59 \%\right)$. Solubilidad en agua: $6.77 \times 10^{-4} \mathrm{~mol} \mathrm{~L}^{-1}$. 
$\left[\mathrm{Sm}(\mathrm{L3})_{3}\right] \cdot 2 \mathrm{H}_{2} \mathrm{O}(9)$

Sólido blanco. Rendimiento 98.8 \% (988.0 mg). Fórmula molecular $\mathrm{C}_{30} \mathrm{H}_{31} \mathrm{SmO}_{11}(\mathrm{PM}=717.93$ $\mathrm{g} / \mathrm{mol}$ ). Análisis elemental: C $49.67 \%$ (C $\mathrm{C}_{\text {teor }} 50.19 \%$ ); H $4.23 \%$ (4.35\%); Sm $23.64 \%$ (24.51\%). IR (ATR, $\left.\mathrm{cm}^{-1}\right): 3525.88 \mathrm{w} ; 2956.87 \mathrm{w} ; 2908.65 \mathrm{w} ; 2839.22 \mathrm{w} ; 1633.71 \mathrm{~s} ; 1604.77 \mathrm{~s} ; 1573.91 \mathrm{~s} ;$ $1560.41 \mathrm{w} ; 1508.33 \mathrm{vs} ; 1460.11 \mathrm{w} ; 1425.40 \mathrm{~s} ; 1384.89 \mathrm{~s} ; 1303.88 \mathrm{w} ; 1240.23 \mathrm{vs} ; 1170.79 \mathrm{vs} ; 1109.07$ w; $1024.20 \mathrm{~s} ; 985.62 \mathrm{~s} ; 883.40 \mathrm{w} ; 831.32 \mathrm{~s} ; 779.24 \mathrm{~s} ; 723.31 \mathrm{~s} ; 696.30 \mathrm{~s} ; 636.51 \mathrm{w}$. TGA pérdida de masa: $4.40 \%\left(30-130{ }^{\circ} \mathrm{C}, 1\right.$ paso, calc. $\left.2 \times \mathrm{H}_{2} \mathrm{O}=5.02 \%\right)$. Solubilidad en agua: $3.83 \times 10^{-4} \mathrm{~mol} \mathrm{~L}^{-1}$.

\section{Evaluación de la actividad catalítica en la oxidación de alquenos}

La oxidación catalítica de los sustratos $\beta$-pineno y canfeno se llevó a cabo utilizando acetonitrilo y tolueno como solventes; como agentes oxidantes se emplearon soluciones de peróxido de hidrógeno (30\%) y ácido peracético (12\%). La mezcla de reacción se preparó disolviendo el catalizador lantánido (0.01 mmol) en el respectivo solvente; una vez disuelto, se adicionó el sustrato (10.0 mmol) $\mathrm{y}$, a continuación, el agente oxidante $(10.0 \mathrm{mmol})$.

\section{Resultados Y discusión}

\section{Caracterización de los compuestos}

En los espectros FT-IR de los ligandos cinamato de sodio (L1), p-clorocinamato de sodio (L2) y $p$-metoxicinamato de sodio ( $L 3)$ se observan las bandas características de los grupos funcionales de los compuestos obtenidos. Entre las bandas más importantes para el ligando L1 (ver figura 2a) se observa una banda de intensidad media en $1640.68 \mathrm{~cm}^{-1}$ asociada a las vibraciones de tensión del enlace $v(\mathrm{C}=\mathrm{C})$. En $1543.05 \mathrm{~cm}^{-1}$ y $1402.25 \mathrm{~cm}^{-1}$ se observan dos bandas intensas correspondientes a las vibraciones de tensión asimétrica y simétrica del enlace $v\left(\mathrm{COO}^{-}\right)$, respectivamente. Para las vibraciones de deformación fuera del plano del enlace $\gamma(=\mathrm{CH})$ aparece una banda intensa en $968.27 \mathrm{~cm}^{-1}$, que es característica de la sustitución trans en alquenos (Kalinowska, Lewandowski, Swisłocka y Regulska, 2010).
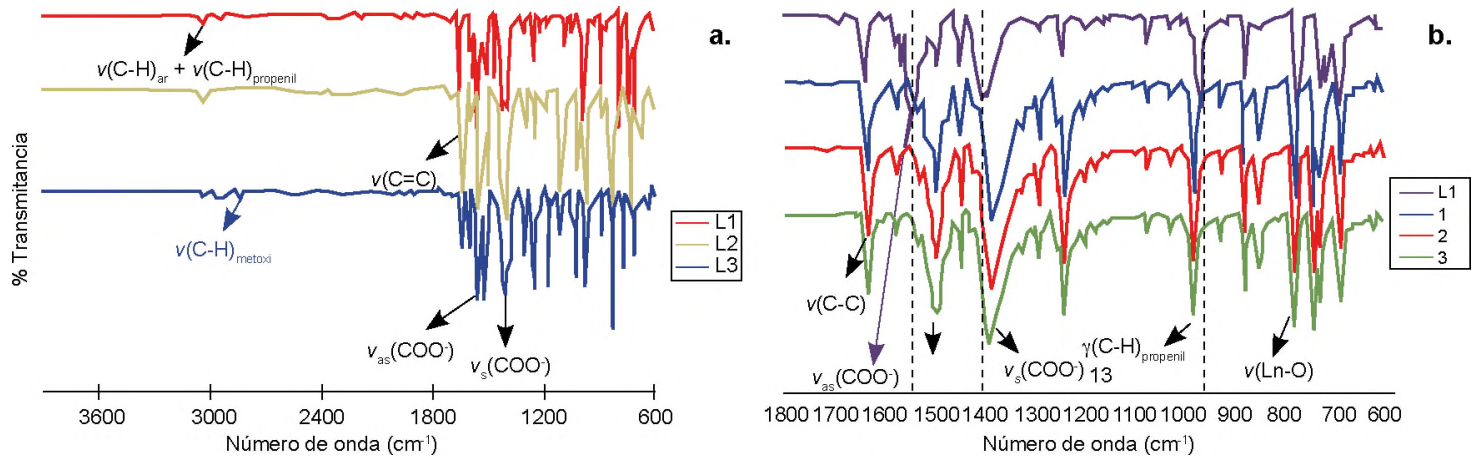

Figura 2. Espectros FT-IR: a) los ligandos cinamato de sodio ( $L 1)$, p-clorocinamato de sodio ( $L 2)$ y $p$-metoxicinamato de sodio ( $L 3)$ y b) ligando cinamato de sodio ( $L 1)$ y los complejos $\left[\mathrm{Ce}(\mathrm{L} 1)_{3} \cdot 0.5 \mathrm{H}_{2} \mathrm{O}\right](1)$, $\left[\mathrm{Nd}(\mathrm{L} 1)_{3} \cdot 0.4 \mathrm{H}_{2} \mathrm{O}\right](2)$ y $\left[\mathrm{Sm}(\mathrm{L} 1)_{3} \cdot 0.5 \mathrm{H}_{2} \mathrm{O}\right](3)$

En los ligandos $L 2$ y $L 3$ se observan unas frecuencias similares a las del espectro del ligando L1; sin embargo, se encuentran algunas diferencias significativas, como la presencia de una banda en $2839.22 \mathrm{~cm}^{-1}$ en el espectro del ligando $L 3$, asociada a las vibraciones de tensión del enlace $v(\mathrm{C}-\mathrm{H})$ 
del grupo $-\mathrm{OCH}_{3}$. Además, en el espectro del ligando $L 2$ y $L 3$ aparece una banda intensa en 829.39 $\mathrm{cm}^{-1} \mathrm{y}$ en $825.53 \mathrm{~cm}^{-1}$ correspondiente a la vibración de deformación fuera del plano de los enlaces $\gamma(\mathrm{C}=\mathrm{C})$, bandas que son características de los anillos aromáticos en posición 1.4-disustituidos. Finalmente, en el espectro del ligando $L 3$ se observan dos bandas asociadas a la vibración de tensión del enlace $v(\mathrm{C}-\mathrm{O}-\mathrm{C})$ del grupo metoxi localizadas a 1240.23 y $1029.99 \mathrm{~cm}^{-1}$. En la figura $2 \mathrm{~b}$ se muestran los espectros FT-IR (expansión en la región de la huella dactilar) del ligando L1 y de los complejos sintetizados a partir de este. El análisis de los espectros infrarrojo de los otros complejos es análogo y se muestra en la información suplementaria. En el análisis de L1 y los complejos se determinó la diferencia entre los valores de número de onda de las bandas de vibración de tensión asimétrica y simétrica de los grupos carboxilato, con el objetivo de distinguir entre las tres formas de unión de los complejos con iones carboxilato, unidentada, bidentada o quelato y unión puente (Deacon, Huber y Phillips, 1980). Cabe resaltar que para los complejos sintetizados los valores de $\Delta v\left(\mathrm{CO}_{2}^{-}\right)$se ajustan al tipo de unión bidentada o quelato (ver tabla 1). Por otro lado, se observó una banda intensa entre $730-783 \mathrm{~cm}^{-1} \mathrm{co}-$ rrespondiente a la vibración de tensión del enlace metal-oxígeno $v(\mathrm{Ln}-\mathrm{O})$, lo que también confirma la coordinación del oxígeno al centro metálico (Aragón-Muriel y Polo-Cerón, 2013).

Tabla 1. Valores experimentales de las vibraciones observadas en los espectros FT-IR para los ligandos y complejos sintetizados

\begin{tabular}{|c|c|c|c|c|c|c|}
\hline \multirow{2}{*}{ Compuesto } & \multicolumn{6}{|c|}{ Bandas características (cm-1) } \\
\hline & $v(\mathrm{OH})$ & $v(\mathbf{C}=\mathrm{C})$ & $v_{a s}($ CO2- $)$ & $v_{s}(\mathrm{CO} 2-)$ & $\Delta \mathrm{n}(\mathrm{CO} 2-)^{*}$ & $v($ Ln-O) \\
\hline L1 & -- & 1639.49 & 1543.05 & 1402.25 & 140.80 & -- \\
\hline 1 & -- & 1633.71 & 1490.97 & 1384.89 & 106.08 & 777.31 \\
\hline 2 & -- & 1633.71 & 1490.97 & 1386.82 & 104.15 & 779.24 \\
\hline 3 & -- & 1633.71 & 1490.97 & 1390.68 & 100.29 & 779.24 \\
\hline L2 & -- & 1641.42 & 1539.20 & 1398.39 & 140.81 & -- \\
\hline 4 & 3369.64 & 1635.64 & 1489.05 & 1386.82 & 102.23 & 736.81 \\
\hline 5 & -- & 1635.64 & 1489.05 & 1384.89 & 104.16 & 736.81 \\
\hline 6 & 3604.96 & 1635.64 & 1489.05 & 1379.10 & 109.95 & 742.59 \\
\hline L3 & -- & 1639.49 & 1546.91 & 1406.11 & 140.80 & -- \\
\hline 7 & 3502.73 & 1631.78 & 1508.33 & 1386.82 & 121.51 & 779.24 \\
\hline 8 & 3562.52 & 1631.78 & 1508.33 & 1388.75 & 119.58 & 779.24 \\
\hline 9 & 3525.88 & 1635.63 & 1508.33 & 1384.89 & 123.44 & 782.33 \\
\hline
\end{tabular}

${ }^{*} \Delta v\left(\mathrm{CO}_{2}\right)=\left[v_{a s}\left(\mathrm{COO}^{-}\right)-v_{s}\left(\mathrm{COO}^{-}\right)\right]$

En la tabla 1 se puede observar una diferencia entre los valores de $\Delta v\left(\mathrm{CO}_{2}\right)$ de los ligandos y los complejos, esto se debe a que los iones metálicos afectan la estructura molecular de los ligandos $p-R$-cinamato, dado que influyen en la distribución de la carga electrónica del anión carboxilato y en el doble enlace entre el anillo aromático y el grupo carboxilato (Kalinowska et ál., 2010).

Para los análisis termogravimétricos, como ejemplo representativo se muestran las curvas de TG-DTG de los complejos 1, 2 y 3 (ver figura 3). Las curvas TG-DTG de los complejos 4-9 se encuentran en la información suplementaria. En los complejos 1, 2 y 3 se observan pérdidas de masa en tres pasos. El primer paso, entre $280-340^{\circ} \mathrm{C}$, presenta una pérdida de masa correspondiente a las moléculas de agua que pueden estar presentes como ligandos al centro metálico en la red polimérica de los complejos; estos resultados concuerdan con lo observado en compuestos similares reportados con 0.4 moléculas de agua (Carvalho, Fernandes, Fertonani y Ionashiro 2003). En el segundo, entre $330-590^{\circ} \mathrm{C}$, se observan dos pasos consecutivos correspondientes a la descomposición térmica de los complejos anhidros con la formación de los respectivos carbonatos 
y posteriormente óxidos. Las temperaturas, las pérdidas de masa y los productos formados en cada paso para los complejos 1-9 se reportan en la tabla 2.
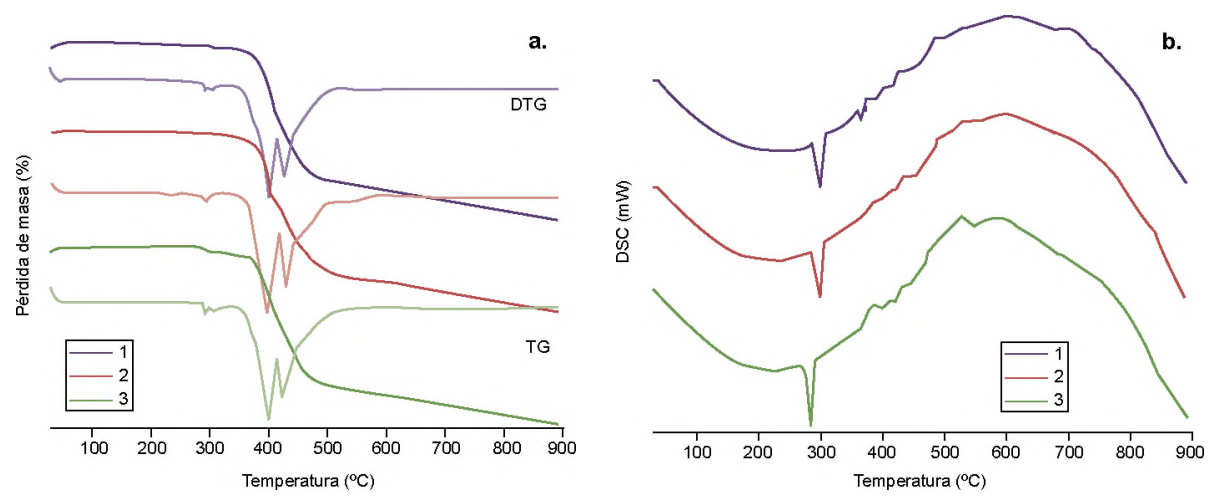

Figura 3. Estudios térmicos de los complejos [Ce(L1) $\left.)_{3} \cdot 0.5 \mathrm{H}_{2} \mathrm{O}\right](1),\left[\mathrm{Nd}(\mathrm{L} 1)_{3} \cdot 0.4 \mathrm{H}_{2} \mathrm{O}\right](2),\left[\mathrm{Sm}(\mathrm{L} 1)_{3} \cdot 0.5 \mathrm{H}_{2} \mathrm{O}\right]$ (3): a) curvas TG-DTG, b) curvas DSC.

Tabla 2. Valores experimentales de temperatura y porcentaje de pérdida de masa, observados en cada paso de las curvas TG- DTG en atmósfera inerte

\begin{tabular}{|c|c|c|c|c|}
\hline \multirow{2}{*}{ Compuesto } & \multicolumn{3}{|c|}{ Temperatura / pérdida de masa $\left[\mathrm{T}\left({ }^{\circ} \mathrm{C}\right) / \Delta \mathrm{m}(\%)\right]$} & \multirow{2}{*}{ Productos obtenidos } \\
\hline & Primer paso & Segundo paso & Tercer paso & \\
\hline 1 & $\begin{array}{l}280-340 / 1.59 \\
\text { (calc. } 1.53 \% \text { ) }\end{array}$ & $\begin{array}{l}340-410 / 20.25 \\
\text { (calc. } 20.86 \% \text { ) }\end{array}$ & $\begin{array}{l}410-590 / 28.62 \\
\text { (calc. } 28.68 \% \text { ) }\end{array}$ & $\begin{array}{l}\mathrm{Ce}(\mathrm{L} 1)_{3} \\
\mathrm{Ce}_{2}\left(\mathrm{CO}_{3}\right)_{3} \\
\mathrm{Ce}_{2} \mathrm{O}_{3}\end{array}$ \\
\hline 2 & $\begin{array}{l}280-330 / 1.17 \\
\text { (calc. } 1.22 \% \text { ) }\end{array}$ & $\begin{array}{l}330-420 / 20.24 \\
\text { (calc. } 20.01 \% \text { ) }\end{array}$ & $\begin{array}{l}420-570 / 29.09 \\
\text { (calc. } 28.18 \% \text { ) }\end{array}$ & $\begin{array}{l}\mathrm{Nd}(\mathrm{L} 1)_{3} \\
\mathrm{Nd}_{2}\left(\mathrm{CO}_{3}\right)_{3} \\
\mathrm{Nd}_{2} \mathrm{O}_{3}\end{array}$ \\
\hline 3 & $\begin{array}{l}280-340 / 1.30 \\
\text { (calc. } 1.50 \% \text { ) }\end{array}$ & $\begin{array}{l}370-410 / 19.12 \\
\text { (calc. } 18.89 \% \text { ) }\end{array}$ & $\begin{array}{l}410-580 / 28.10 \\
\text { (calc. } 27.50 \% \text { ) }\end{array}$ & $\begin{array}{l}\mathrm{Sm}(\mathrm{L} 1)_{3} \\
\mathrm{Sm}_{2}\left(\mathrm{CO}_{3}\right)_{3} \\
\mathrm{Sm}_{2} \mathrm{O}_{3}\end{array}$ \\
\hline 4 & $\begin{array}{l}280-330 / 2.76 \\
\text { (calc. } 2.56 \% \text { ) }\end{array}$ & $\begin{array}{l}340-410 / 15.47 \\
\text { (calc. } 15.09 \% \text { ) }\end{array}$ & $\begin{array}{l}410-600 / 20.60 \\
\text { (calc. } 20.86 \% \text { ) }\end{array}$ & $\begin{array}{l}\mathrm{Ce}(\mathrm{L} 2)_{3} \\
\mathrm{Ce}(\mathrm{L} 1)_{3} \\
\mathrm{Ce}_{2}\left(\mathrm{CO}_{3}\right)_{3}\end{array}$ \\
\hline 5 & $\begin{array}{l}210-680 / 44.21 \\
\text { (calc. } 44.78 \% \text { ) }\end{array}$ & -- & -- & $\mathrm{Nd}_{2} \mathrm{O}_{2} \mathrm{CO}_{3}$ \\
\hline 6 & $\begin{array}{l}100-280 / 4.87 \\
\text { (calc } 4.92 \% \text { ) }\end{array}$ & $\begin{array}{l}360-420 / 14.56 \\
\text { (calc } 14.86 \% \text { ) }\end{array}$ & $\begin{array}{l}420-570 / 18.73 \\
\text { (calc. } 18.89 \% \text { ) }\end{array}$ & $\begin{array}{l}\mathrm{Sm}(\mathrm{L} 2)_{3} \\
\mathrm{Sm}(\mathrm{L} 1)_{3} \\
\mathrm{Sm}_{2}\left(\mathrm{CO}_{3}\right)_{3}\end{array}$ \\
\hline 7 & $\begin{array}{l}30-190 / 2.56 \\
\text { (calc. } 2.54 \% \text { ) }\end{array}$ & $\begin{array}{l}190-320 / 2.30 \\
\text { (calc. } 2.54 \% \text { ) }\end{array}$ & $\begin{array}{l}320-460 / 31.50 \\
\text { (calc. } 31.47 \% \text { ) }\end{array}$ & $\begin{array}{l}\mathrm{Ce}(\mathrm{L} 3)_{3} \\
\mathrm{Ce}_{2}\left(\mathrm{CO}_{3}\right)_{3}\end{array}$ \\
\hline 8 & $\begin{array}{l}30-140 / 2.63 \\
\text { (calc. } 2.59 \% \text { ) }\end{array}$ & $\begin{array}{l}350-400 / 13.08 \\
\text { (calc. } 13.33 \% \text { ) }\end{array}$ & $\begin{array}{l}400-490 / 19.16 \\
\text { (calc. } 20.01 \% \text { ) }\end{array}$ & $\begin{array}{l}\mathrm{Nd}(\mathrm{L} 3)_{3} \\
\mathrm{Nd}(\mathrm{L} 1)_{3} \\
\mathrm{Nd}_{2}\left(\mathrm{CO}_{3}\right)_{3}\end{array}$ \\
\hline 9 & $\begin{array}{l}30-130 / 4.40 \\
\text { (calc. } 5.02 \% \text { ) }\end{array}$ & $\begin{array}{l}350-400 / 13.34 \\
\text { (calc. } 13.21 \% \text { ) }\end{array}$ & $\begin{array}{l}400-500 / 19.66 \\
\text { (calc. } 18.89 \% \text { ) }\end{array}$ & $\begin{array}{l}\mathrm{Sm}(\mathrm{L} 3)_{3} \\
\mathrm{Sm}(\mathrm{L} 1)_{3} \\
\mathrm{Sm}_{2}\left(\mathrm{CO}_{3}\right)_{3}\end{array}$ \\
\hline
\end{tabular}


Para el complejo 4 la pérdida de masa ocurre en tres pasos. El primer paso $\left(280-330^{\circ} \mathrm{C}\right)$ es debido a la pérdida de una molécula de $\mathrm{H}_{2} \mathrm{O}$; el segundo $\left(340-410^{\circ} \mathrm{C}\right)$, corresponde a la formación de $\mathrm{Ce}(\mathrm{L} 1)_{3}$, y el último $\left(410-600^{\circ} \mathrm{C}\right)$, es causado por la descomposición para la formación de $\mathrm{Ce}_{2}\left(\mathrm{CO}_{3}\right)_{3}$. Para el complejo 5 no se observan pérdidas de masa de hidratación; el único paso es hacia la formación del dioxicarbonato de neodimio (Mercandante et ál., 1993). Para el complejo 6 el primer paso es la deshidratación de dos moléculas de $\mathrm{H}_{2} \mathrm{O}$. En los dos últimos pasos se forma el compuesto $\mathrm{Sm}(\mathrm{L} 1)_{3}$, que finalmente se descompone a $\mathrm{Sm}_{2}\left(\mathrm{CO}_{3}\right)_{3}$. Para los complejos 7 y 9 se observa evaporación de dos moléculas de agua, respectivamente, mientras que para el complejo 8 solo se pierde una molécula de agua.

En las curvas DSC de los complejos 1-3 (figura 3b) se observan los picos endotérmicos atribuidos a la fusión y la descomposición térmica de los compuestos. Para los complejos 1-2 el primer pico endotérmico a $298^{\circ} \mathrm{C}$ se atribuye a la temperatura de fusión; en el complejo 3, se observa a $283^{\circ} \mathrm{C}$. Después de la fusión, se evidencian otros eventos térmicos entre 366 a $485^{\circ} \mathrm{C}$ para el complejo 1 , entre 430 a $490^{\circ} \mathrm{C}$ para el complejo 2 y entre $350-550^{\circ} \mathrm{C}$ para el complejo 3 , atribuidos a la descomposición térmica de los cinamatos.

Para el complejo 4 se observa un pico exotérmico y un pico endotérmico que puede ser debido a procesos de cristalización y descomposición térmica, respectivamente. Los picos endotérmicos entre $100-150^{\circ} \mathrm{C}$ observados en las curvas DSC de los complejos 6-9 corresponden a la pérdida de agua, como se observó en las curvas TG-DTG. Los picos endotérmicos entre $200-280^{\circ} \mathrm{C}$ evidenciados en las curvas DSC de los complejos $8-9$ se atribuyen a la temperatura de fusión, mientras que los picos exotérmicos registrados entre $450-480^{\circ} \mathrm{C}$ pueden ser causados por la oxidación de los compuestos anhidro de las etapas anteriores. Las curvas DSC de los complejos 4-9 se encuentran en la información suplementaria.

\section{Resultados de actividad catalítica}

Para la oxidación catalítica del $\beta$-pineno y el canfeno, se evaluaron las condiciones de reacción más favorables para obtener el epóxido como uno de los productos. La interpretación de los resultados obtenidos se llevó a cabo mediante la determinación de los porcentajes de conversión y selectividad. Para el análisis de los productos de oxidación del $\beta$-pineno, se debe tener en cuenta la estructura del compuesto. Este adopta preferiblemente una conformación pseudo-silla, en la que el hidrógeno alílico es aproximadamente ortogonal al doble enlace y, por lo tanto, es más fácilmente extraíble; luego, la oxidación alílica es la ruta más probable para la oxidación de este monoterpeno (Pakdel, Sarron y Roy, 2001). Cabe resaltar que la oxidación alílica es un proceso que implica radicales libres y es más probable en presencia de especies metálicas, que forman un intermediario de bajo estado de oxidación. Para la oxidación catalítica del $\beta$-pineno se evaluó el efecto de la temperatura ( $35 \mathrm{y}$ $\left.70{ }^{\circ} \mathrm{C}\right)$, la relación catalizador:sustrato (1:500 y 1:1000), la influencia del agente oxidante $\left(\mathrm{H}_{2} \mathrm{O}_{2}\right.$ y $\mathrm{CH}_{3} \mathrm{CO}_{2} \mathrm{OH}$ ) y el efecto del solvente (acetonitrilo y tolueno). Inicialmente se realizó la oxidación del $\beta$-pineno a $35^{\circ} \mathrm{C}$ en acetonitrilo y utilizando peróxido de hidrógeno. En este caso se empleó una relación catalizador:sustrato (1:500). A esta temperatura se obtuvo una baja conversión y solo se observó en algunos catalizadores la formación de mirtenol y mirtenal en muy bajas cantidades. Posteriormente, se realizó la oxidación de $\beta$-pineno a $70^{\circ} \mathrm{C}$ utilizando tolueno como solvente, ácido peracético como agente oxidante y una relación catalizador:sustrato 1:1000; bajo estas condiciones, se observó la formación del epóxido de $\beta$-pineno, acompañado de una serie de productos (ver figura 4). Los espectros de masas de los compuestos obtenidos se encuentran en la información suplementaria. En la tabla 3 se observa que con el complejo de $\mathrm{Ce}$ (III) (7) se presentó la conversión más alta (82.62\%) con una selectividad del $70.52 \%$ hacia el producto 1 (nopinona), que hace parte de diversos aceites esenciales empleados en la industria de química fina. Con los 
demás catalizadores se obtuvieron conversiones entre 36 y $81 \%$. Acerca de la selectividad, se puede ver claramente que el $\beta$-pineno se convirtió principalmente en nopinona.

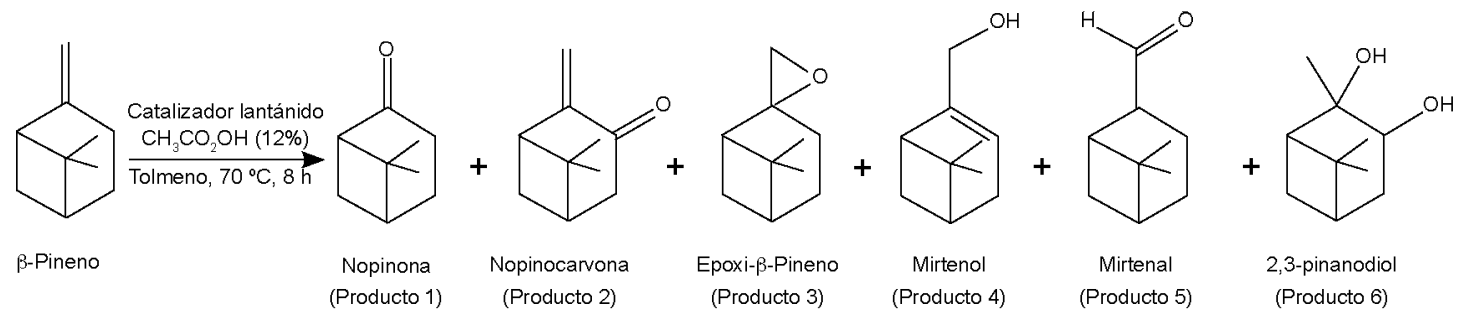

Figura 4. Productos de oxidación de $\beta$-pineno en tolueno, utilizando ácido peracético como agente oxidante.

Tabla 3. Porcentajes de conversión y selectividad hacia los productos obtenidos en la oxidación de $\beta$-pineno utilizando tolueno y ácido peracético

\begin{tabular}{cccccccc}
\hline \multirow{2}{*}{ Catalizador } & $\begin{array}{c}\text { Conversión } \\
(\%)\end{array}$ & \multicolumn{7}{c}{ Selectividad (\%) } \\
\cline { 3 - 8 } & Producto 1 & Producto 2 & Producto 3 & Producto 4 & Producto 5 & Producto 6 \\
\hline 1 & 49.73 & 44.99 & 0.51 & 17.45 & 8.99 & 20.31 & 7.74 \\
2 & 66.99 & 57.61 & 0.13 & 16.88 & 3.73 & 13.72 & 7.93 \\
3 & 81.34 & 64.38 & 0.00 & 8.08 & 1.16 & 11.06 & 15.31 \\
4 & 71.24 & 56.61 & 0.37 & 13.96 & 2.58 & 18.33 & 8.15 \\
5 & 69.16 & 62.28 & 0.32 & 10.42 & 2.34 & 10.56 & 14.07 \\
6 & 52.21 & 55.86 & 0.00 & 14.95 & 3.63 & 15.95 & 9.60 \\
7 & 82.62 & 70.52 & 0.65 & 8.74 & 2.55 & 0.00 & 17.54 \\
8 & 75.99 & 68.07 & 0.79 & 12.59 & 3.10 & 3.39 & 12.04 \\
9 & 36.16 & 61.16 & 0.57 & 10.04 & 8.21 & 13.66 & 6.35 \\
\hline
\end{tabular}

Tiempo: 8-10 h; Temperatura: $70^{\circ} \mathrm{C}$; solvente: tolueno; cat:sust (1:1000); oxidante: $\mathrm{CH}_{3} \mathrm{CO}_{2} \mathrm{OH}$.

Según los resultados obtenidos, se puede inferir que los catalizadores favorecen los productos de oxidación alílicos sobre los epóxidos. Es importante resaltar que la formación de epóxidos utilizando catalizadores ácidos es un reto, debido a que bajo estas condiciones sufren rearreglos y reacciones de isomerización (Suh, Kim, Ahn y Rhee, 2003).

Por otro lado, se realizó la oxidación de $\beta$-pineno a $70^{\circ} \mathrm{C}$ utilizando acetonitrilo como solvente, peróxido de hidrógeno como agente oxidante y una relación catalizador:sustrato (1:1000). Con estas condiciones se observó una disminución en la selectividad hacia el epóxido. No se observó la formación de 2.3-pinanodiol (ver figura 5 y tabla 4). Los espectros de masas de los compuestos obtenidos se encuentran en la información suplementaria. 


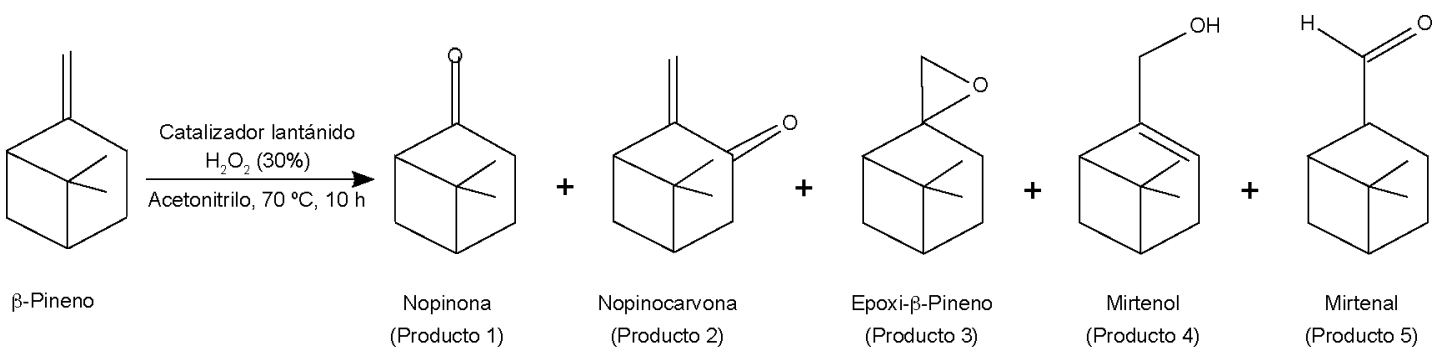

Figura 5. Productos de oxidación de $\beta$-pineno en acetonitrilo, utilizando $\mathrm{H}_{2} \mathrm{O}_{2}$ como agente oxidante.

Tabla 4. Porcentajes de conversión y selectividad hacia los productos obtenidos en la oxidación de $\beta$-pineno utilizando acetonitrilo y peróxido de hidrógeno

\begin{tabular}{ccccccc}
\hline \multirow{2}{*}{ Catalizador } & Conversión (\%) & \multicolumn{5}{c}{ Selectividad (\%) } \\
\cline { 3 - 7 } & & Producto 1 & Producto 2 & Producto 3 & Producto 4 & Producto 5 \\
\hline 1 & 50.01 & 9.64 & 5.54 & 0.00 & 58.34 & 26.47 \\
2 & 84.40 & 12.56 & 5.39 & 0.00 & 69.26 & 12.75 \\
3 & 62.62 & 17.05 & 5.09 & 0.00 & 65.36 & 12.49 \\
4 & 52.90 & 10.19 & 5.96 & 0.00 & 64.47 & 19.37 \\
5 & 72.15 & 15.65 & 6.81 & 0.27 & 63.86 & 13.39 \\
6 & 61.02 & 18.34 & 5.62 & 0.00 & 63.38 & 12.65 \\
7 & 67.36 & 7.65 & 4.70 & 0.00 & 68.79 & 18.83 \\
8 & 57.69 & 0.00 & 6.18 & 0.00 & 79.81 & 14.00 \\
9 & 69.42 & 14.55 & 26.24 & 1.28 & 48.44 & 9.47 \\
\hline
\end{tabular}

Tiempo: 8-10 h; Temperatura: $70{ }^{\circ} \mathrm{C}$; solvente: acetonitrilo; cat:sust (1:1000); oxidante: $\mathrm{H}_{2} \mathrm{O}_{2}$.

Se observó que con el complejo de $\mathrm{Nd}$ (III) (2) se presentó la conversión más alta (84.40\%) con una selectividad del $69.26 \%$ hacia el producto 4 (mirtenol), que es un monoterpeno encontrado en diversas plantas aromáticas con conocida actividad sobre el sistema nervioso central, por lo que es un producto muy interesante para la industria farmacéutica. En cuanto a los demás catalizadores, se observaron conversiones entre 48-72\%. Para todos los complejos, el producto principal fue el producto 4 con selectividades que superaron el $48 \%$. En la figura 6 se observa la gráfica de los porcentajes de conversión a diferentes tiempos utilizando todos los catalizadores. 


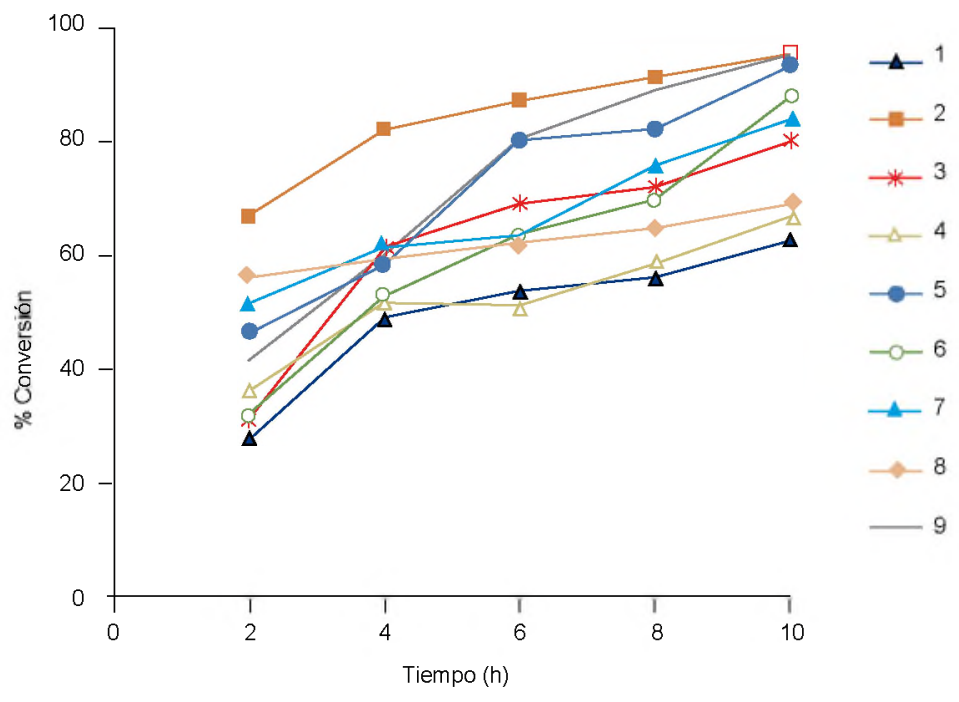

Figura 6. Porcentajes de conversión en la oxidación de $\beta$-pineno a diferentes tiempos con relación 1:1000 (catalizador: $\beta$-pineno), 1:1 $\left(\mathrm{H}_{2} \mathrm{O}_{2}: \beta\right.$-pineno) a $70^{\circ} \mathrm{C}$.

Cuando se utilizó tolueno como solvente y ácido peracético como oxidante, se observó que la selectividad hacia el epóxido fue superior en comparación con la reacción de oxidación en acetonitrilo y peróxido de hidrógeno, mientras que en estas últimas condiciones la selectividad hacia el mirtanal y mirtenol fue mayor (alcanzó selectividades superiores al $48 \%$ para el mirtanal). En cuanto a la conversión, se vio una disminución en condiciones ácidas acompañadas de solventes poco polares, mientras que en solventes más polares y en peróxido de hidrógeno la conversión fue mayor. Por otro lado, se puede mencionar que no se evidenció una influencia significativa del metal ni del sustituyente sobre la conversión y selectividad hacia los productos.

En el caso del canfeno -a diferencia del $\beta$-pineno- se puede observar que este sólo tiene un hidrógeno alílico, el cual es difícil de extraer; luego, la oxidación alílica no es la ruta más probable para este monoterpeno. Inicialmente, se realizó la reacción de oxidación a $70{ }^{\circ} \mathrm{C}$ utilizando $\mathrm{H}_{2} \mathrm{O}_{2}$ como agente oxidante y tolueno como solvente; sin embargo, la conversión no superó el 7 \% con ninguno de los catalizadores. En vista de lo anterior, se utilizó ácido peracético como agente oxidante manteniendo las mismas condiciones. Se observó que a pesar de que el ácido peracético es utilizado frecuentemente para la epoxidación de olefinas, en este caso no se evidenció la formación del epóxido de canfeno, en su lugar se obtuvieron cetonas, alcoholes, ácidos carboxílicos y glicoles (ver figura 7). Los espectros de masas de los compuestos obtenidos se encuentran en la información suplementaria.

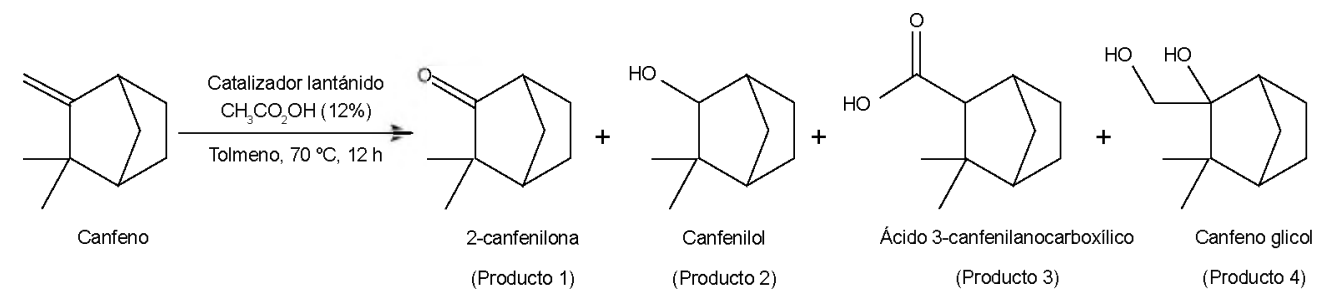

Figura 7. Principales productos de oxidación de canfeno en tolueno, utilizando ácido peracético como agente oxidante. 
En estas condiciones se observó que el complejo de $\mathrm{Nd}(\mathrm{III})$ (2) presentó la conversión más alta (97.99\%) con una selectividad del $84.05 \%$ hacia el producto 3 (ácido 3-canfenilanocarbóxilico), que forma parte de plantas aromáticas empleadas en la industria farmacéutica. En cuanto a los demás catalizadores, se observaron conversiones relativamente altas (86-97\%). Para los complejos 3-8 el producto principal fue 2-canfenilona, mientras que para los complejos 1 y 2 el producto principal fue ácido 3-canfenilocarboxílico (ver tabla 5 y figura 8 ).

Tabla 5. Porcentajes de conversión y selectividad hacia los productos obtenidos en la oxidación de canfeno

\begin{tabular}{ccccccc}
\hline \multirow{2}{*}{ Catalizador } & Conversión (\%) & \multicolumn{5}{c}{ Selectividad (\%) } \\
\cline { 3 - 7 } & & Producto 1 & Producto 2 & Producto 3 & Producto 4 & $\begin{array}{c}\text { Productos de } \\
\text { isomerización }\end{array}$ \\
\hline 1 & 91.89 & 13.94 & 1.73 & 77.14 & 4.39 & 2.79 \\
2 & 97.99 & 7.77 & 1.25 & 84.05 & 4.56 & 2.37 \\
3 & 90.12 & 67.90 & 18.40 & 6.78 & 6.91 & 0.00 \\
4 & 86.90 & 77.87 & 17.02 & 4.22 & 0.88 & 0.00 \\
5 & 91.74 & 46.84 & 11.71 & 12.19 & 28.09 & 1.17 \\
6 & 90.95 & 61.58 & 6.59 & 9.29 & 21.99 & 0.54 \\
7 & 86.96 & 61.44 & 6.88 & 19.04 & 10.12 & 2.52 \\
8 & 97.81 & 60.28 & 11.97 & 13.91 & 13.83 & 0.00 \\
\hline
\end{tabular}

Tiempo: $12 \mathrm{~h}$, Temperatura: $70^{\circ} \mathrm{C}$, solvente: tolueno, cat:sust (1:1000), oxidante: $\mathrm{CH}_{3} \mathrm{CO}_{2} \mathrm{OH}$.

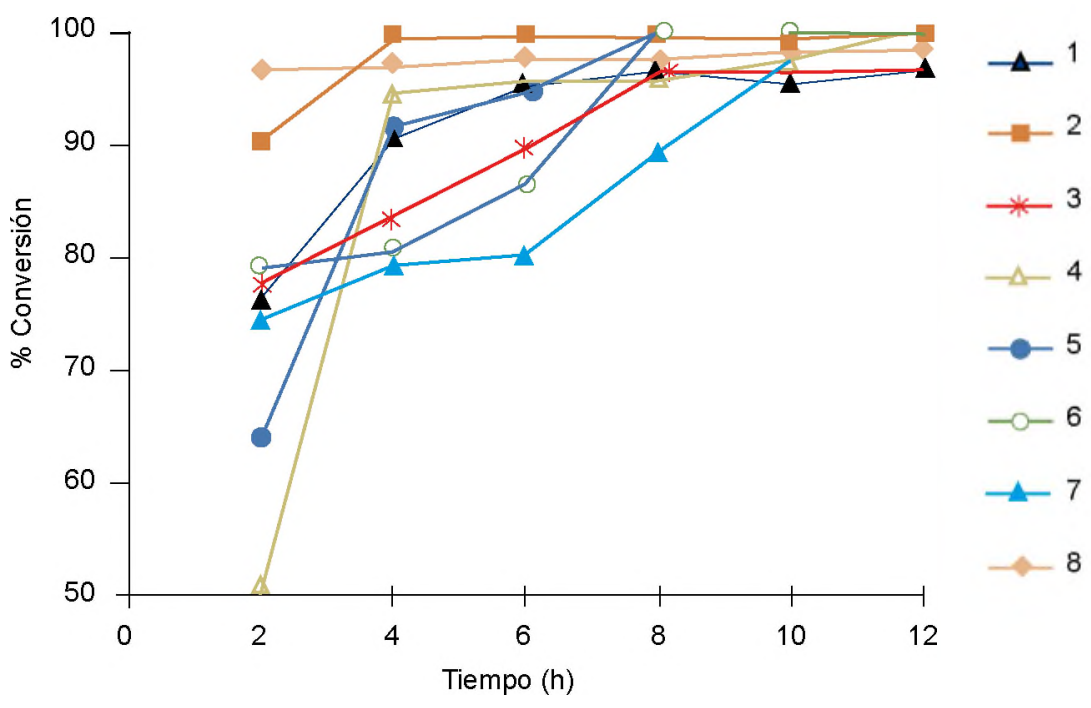

Figura 8. Porcentajes de conversión en la oxidación de canfeno a diferentes tiempos con relación 1:1000 (catalizador: canfeno), 1:1 (ácido peracético: canfeno) a $70^{\circ} \mathrm{C}$.

Uno de los factores por los cuales se obtuvo una baja conversión de los productos en la oxidación de canfeno con peróxido de hidrógeno puede ser la concentración del agente oxidante. Encontrar la concentración óptima es de gran importancia, ya que una baja concentración puede reflejar una baja actividad del catalizador, mientras que un exceso de oxidante puede conducir a la formación 
de muchos subproductos; sin embargo, la influencia de la concentración del agente oxidante no fue evaluada en este ensayo preliminar. Por otro lado, la utilización de ácido peracético condujo a la formación de varios productos; no obstante, la obtención del epóxido de canfeno no fue posible. Una de las razones por las cuales la formación del epóxido fue muy poco probable o poco detectable es la inestabilidad del producto, ya que en presencia de agua (subproducto del agente oxidante) y catalizada por la acidez del catalizador el anillo epoxi se rompe, lo que da como resultado la formación de canfeno glicol (producto 4). Además, se observó la formación de aldehído canfolénico, un producto de isomerización del epóxido de canfeno (Patel, 2013; Cejka, Corma y Zones, 2010). Cabe resaltar que este compuesto no fue detectado en ausencia del catalizador.

\section{Conclusiones}

Se realizó la síntesis de nueve complejos lantánidos a partir de los nitratos de cerio, neodimio y samario y derivados de los ácidos trans-cinámico, trans-p-clorocinámico y trans-p-metoxicinámico. Las mediciones de FT-IR indicaron que los complejos se ajustan al tipo de unión bidentada o quelato. El análisis térmico simultáneo proporcionó información importante relacionada con la descomposición térmica, además de la presencia de moléculas de agua de deshidratación y de coordinación, así como los puntos de fusión de la mayoría de complejos. Los resultados obtenidos en cada una de las técnicas concuerdan con las estructuras propuestas para cada uno de los compuestos sintetizados. Finalmente, se evaluó la actividad catalítica de los complejos en la oxidación de $\beta$-pineno y canfeno. En la oxidación de $\beta$-pineno con ácido peracético, se observó que con el complejo $\left[\mathrm{Ce}(\mathrm{L} 3)_{3} \cdot \mathrm{H}_{2} \mathrm{O}\right] \cdot \mathrm{H}_{2} \mathrm{O}(7)$ se presentó la conversión más alta $(82.62 \%)$ con una selectividad del $70.52 \%$ hacia la nopinona, mientras que con peróxido de hidrógeno se advirtió que con el complejo $\left[\mathrm{Nd}(\mathrm{L} 1)_{3} \cdot 0.4 \mathrm{H}_{2} \mathrm{O}\right](2)$ se presentó la mayor actividad con una conversión del $84.40 \%$ y una selectividad del $69.26 \%$ hacia el mirtenol. Se encontró que la reacción de oxidación de $\beta$-pineno a $35^{\circ} \mathrm{C}$ con estos catalizadores y bajo las condiciones empleadas conduce a bajas conversiones. Este mismo resultado se evidenció durante la reacción de oxidación de canfeno en tolueno y utilizando peróxido de hidrógeno como agente oxidante. Por otro lado, se encontró que empleando tolueno como solvente y ácido peracético como agente oxidante se favorece la formación del epóxido de $\beta$-pineno, mientras que en estas mismas condiciones no se obtuvo el epóxido de canfeno; sin embargo, estas condiciones favorecieron la formación de mirtanal y mirtenol, que alcanzó selectividades superiores al $48 \%$ para el mirtanal.

\section{Referencias}

Aragón-Muriel, A., Camprubi, M., Gonzalez, E., Salinas, A., Rodriguez, A., Gomez, S., y PoloCerón, D. (2014). Dual investigation of lanthanide complexes with cinnamate and phenylacetate ligands: study of the cytotoxic properties and the catalytic oxidation of styrene. Polybedron, 80, 117-128.

Aragón-Muriel, A., y Polo-Cerón, D. (2013). Synthesis, characterization, thermal behavior, and antifungal activity of $\mathrm{La}$ (III) complexes with cinnamates and 4-methoxyphenylacetate. Journal of Rare Earths, 31(11), 1106-1113.

Aragón-Muriel, A., Upegui, Y., Muñoz, J., Robledo, S., y Polo-Cerón, D. (2016). Synthesis, characterization and biological evaluation of rare earth complexes against tropical diseases Leishmaniasis, malaria and trypanosomiasis. Avances en Quimica, 11(2), 53-61.

Belanger, J. (1998). Perillyl alcohol: applications in oncology. Alternative Medicine Review, 3(6), 448-457. 
Blaz, E., y Pielichowski, J. (2006). Polymer-Supported Cobalt (II) Catalysts for the Oxidation of Alkenes. Molecules, 11(1), 115-120.

Caovilla, M., Caovilla, A., Pergher, S., Esmelindro, M., Fernandes, C., Dariva, C., Bernardo-Gusmao, K., Oestreicher, E., y Antunes, O. (2008). Catalytic oxidation of limonene, $\alpha$-pinene and $\beta$-pinene by the complex $\left[\mathrm{Fe}^{\mathrm{III}}(\mathrm{BPMP}) \mathrm{Cl}(\mu-\mathrm{O}) \mathrm{Fe}^{\mathrm{III}} \mathrm{Cl}_{3}\right]$ biomimetic to MMO enzyme. Catalysis Today, 133-135, 695-698.

Carvalho, M., Fernandes, N., Fertonani, F. y Ionashiro, M. (2003). A thermal behaviour study of solid-state cinnamates of the latter trivalent lanthanides and yttrium(III). Thermochimica Acta, 398(1), 93-99.

Casuscelli, S., Eimer, G., Canepa, A., Heredia, A., Poncio, C., Crivello, M., Perez, C., Aguilar, A., y Herrero, E. (2008). Ti-MCM-41 as catalyst for $\alpha$-pinene oxidation. Study of the effect of Ti content and $\mathrm{H}_{2} \mathrm{O}_{2}$ addition on activity and selectivity. Catalysis Today, 133-135, 678-683

Cejka, J., Corma, A., y Zones, S. (Eds). (2010). Zeolites and Catalysis: Synthesis, Reactions and Applications. Alemania: John Wiley \& Sons.

Corma, A., Renz, M., y Susarte, M. (2009). Transformation of biomass products into fine chemicals catalyzed by solid Lewis- and Brønsted-acids. Topics in Catalysis, 52(9), 1182-1189.

da Silva, M., Vieira, L., Oliveira, A., y Ribeiro, M. (2013). Novel effect of palladium catalysts on chemoselective oxidation of $\beta$-pinene by hydrogen peroxide. Monatshefte für ChemieChemical Monthly, 144(3), 321-326.

de Oliveira, A., Lopes, M., da Silva, M. (2009). Palladium-Catalysed Oxidation of Bicycle Monoterpenes by Hydrogen Peroxide in Acetonitrile Solutions: A Metal Reoxidant-Free and Environmentally Benign Oxidative Process. Catalysis Letters, 130, 424-431.

Deacon, G., Huber, F., y Phillips R. (1980) Relationships between the carbon-oxygen stretching frequencies of carboxylato complexes and the type of carboxylate coordination. Coordination Chemistry Reviews, 33(3), 227-250.

Kalinowska, M., Lewandowski, W., Swisłocka, R., y Regulska, E. (2010). The FT-IR, FT-Raman, ${ }^{1} \mathrm{H}$ and ${ }^{13} \mathrm{C}$ NMR study on molecular structure of sodium(I), calcium(II), lanthanum(III) and thorium(IV) cinnamates. Journal of Spectroscopy, 24(3-4), 277-281.

Mercandante, A., Ionashiro, M., de Oliveira, L., Ribeiro, C., y Moscardini, L. (1993). Preparation and thermal decomposition of solid state lanthanide(III) and yttrium(III) chelates of ethylenediaminetetraacetic acid. Thermochimica Acta, 216, 267-277.

Nalone, L., Cardoso, T., Guimarães, R., do Ó Pessoa, C., Odorico, M., Marques, B., Gomes, L., Andrade, A., y Pergentino, D. (2015). Evaluation of the cytotoxicity of structurally correlated p-menthane derivatives. Molecules, 20(7), 13264-13280.

Pakdel, H., Sarron, S., y Roy, C. (2001). $\alpha$-Terpineol from hydration of crude sulfate turpentine oil. Journal of Agricultural and Food Chemistry, 49(9), 4337-4341.

Patel, A. (Ed.). (2013). Environmentally benign catalysts: for clean organic reactions. India: Springer Science \& Business Media.

Silva, M., Robles-Dutenhefner, P., Menini, L., y Gusevskaya, E. (2003). Cobalt catalyzed autoxidation of monoterpenes in acetic acid and acetonitrile solutions. Journal of Molecular Catalysis A: Chemical, 201(1), 71-77. 
Stolle, A. (2013). Synthesis of Nopinone from $\beta$-Pinene-A Journey Revisiting Methods for Oxidative Cleavage of $\mathrm{C}=\mathrm{C}$ Bonds in Terpenoid Chemistry. European Journal of Organic Chemistry, 2013(12), 2265-2278.

Suh, Y., Kim, N., Ahn, W., y Rhee, H. K. (2003) One-pot synthesis of campholenic aldehyde from $\alpha$-pinene over Ti-HMS catalyst II: effects of reaction conditions. Journal of Molecular Catalysis A: Chemical, 198(1), 309-316. 
INFORMACIÓN SUPLEMENTARIA

Anexo 1. Abreviaturas y símbolos

\begin{tabular}{|c|c|c|}
\hline Símbolo, sigla o abreviatura & Significado & Nomenclatura adicional \\
\hline L1 & $\begin{array}{l}\text { trans-cinamato, } \\
\text { cinamato de sodio }\end{array}$ & Ligando 1 \\
\hline L2 & $\begin{array}{l}\text { trans-p-clorocinamato, } \\
p \text {-clorocinamato de sodio }\end{array}$ & Ligando 2 \\
\hline L3 & $\begin{array}{l}\text { trans-p-metoxicinamato, } \\
\text { p-metoxicinamato de sodio }\end{array}$ & Ligando 3 \\
\hline$Y(I I I)$ & Itrio en estado de oxidación $3+$ & - \\
\hline $\mathrm{La}(\mathrm{III})$ & Lantano en estado de oxidación $3+$ & - \\
\hline $\mathrm{Ce}(I I I)$ & Cerio en estado de oxidación 3+ & - \\
\hline $\mathrm{Nd}(\mathrm{III})$ & Neodimio en estado de oxidación $3+$ & - \\
\hline $\operatorname{Sm}(I I I)$ & Samario en estado de oxidación 3+ & - \\
\hline $\mathrm{Yb}(\mathrm{III})$ & Iterbio en estado de oxidación $3+$ & - \\
\hline FT-IR & Espectroscopia infrarroja por transformadas de Fourier & IR \\
\hline ATR & Reflectancia total atenuada & - \\
\hline $\mathrm{AE}$ & Análisis Elemental & EA \\
\hline GC-MS & $\begin{array}{l}\text { Cromatografía de gases acoplado a un espectrómetro } \\
\text { de masas }\end{array}$ & - \\
\hline$\% \mathrm{C}$ & Porcentaje de carbono & - \\
\hline$\% \mathrm{H}$ & Porcentaje de hidrógeno & - \\
\hline$\%$ Ln & Porcentaje de lantánido & - \\
\hline EDTA & Ácido etilendiaminotetraacético & - \\
\hline TGA & Análisis termogravimétrico & - \\
\hline DSC & Calorimetría diferencial de barrido & - \\
\hline $\mathrm{NaOH}$ & Hidróxido de sodio & - \\
\hline $\mathrm{Ce}\left(\mathrm{NO}_{3}\right)_{3} \cdot 6 \mathrm{H}_{2} \mathrm{O}$ & Nitrato de cerio hexahidratado & - \\
\hline$\left[\mathrm{Ce}(\mathrm{L} 1)_{3} \cdot 0.5 \mathrm{H}_{2} \mathrm{O}\right]$ & Complejo de cerio con ligando 1 & (1) \\
\hline$\left[\mathrm{Nd}(\mathrm{L} 1)_{3} \cdot 0.4 \mathrm{H}_{2} \mathrm{O}\right]$ & Complejo de neodimio con ligando 1 & $(2)$ \\
\hline$\left[\mathrm{Sm}(\mathrm{L} 1)_{3} \cdot 0.5 \mathrm{H}_{2} \mathrm{O}\right]$ & Complejo de samario con ligando 1 & (3) \\
\hline$\left[\mathrm{Ce}(\mathrm{L} 2)_{3} \cdot \mathrm{H}_{2} \mathrm{O}\right]$ & Complejo de cerio con ligando 2 & $(4)$ \\
\hline$\left[\mathrm{Nd}(\mathrm{L} 2)_{3}\right]$ & Complejo de neodimio con ligando 2 & (5) \\
\hline$\left[\mathrm{Sm}(\mathrm{L} 2)_{3}\right] \cdot 2 \mathrm{H}_{2} \mathrm{O}$ & Complejo de samario con ligando 2 & $(6)$ \\
\hline$\left[\mathrm{Ce}(\mathrm{L} 3)_{3} \cdot \mathrm{H}_{2} \mathrm{O}\right] \cdot \mathrm{H}_{2} \mathrm{O}$ & Complejo de cerio con ligando 3 & $(7)$ \\
\hline$\left[\mathrm{Nd}(\mathrm{L} 3)_{3}\right] \cdot \mathrm{H}_{2} \mathrm{O}$ & Complejo de neodimio con ligando 3 & (8) \\
\hline$\left[\mathrm{Sm}(\mathrm{L} 3)_{3}\right] \cdot 2 \mathrm{H}_{2} \mathrm{O}$ & Complejo de samario con ligando 3 & (9) \\
\hline $\mathrm{H}_{2} \mathrm{O}$ & Agua & - \\
\hline C (en "Análisis elemental") & Porcentaje de Carbono experimental & - \\
\hline $\mathrm{C}_{\text {teor }}$ (en "Análisis elemental") & Porcentaje de Carbono teórico & - \\
\hline w & weak (débil) & - \\
\hline vw & very weak (muy débil) & - \\
\hline s & strong (fuerte) & - \\
\hline vs & very strong (muy fuerte) & - \\
\hline
\end{tabular}




\begin{tabular}{|c|c|c|c|c|c|}
\hline \multicolumn{2}{|c|}{ Símbolo, sigla o abreviatura } & \multicolumn{3}{|c|}{ Significado } & Nomenclatura adicional \\
\hline calc. & & \multicolumn{3}{|c|}{ Calculado } & - \\
\hline$v(C=C)$ & & \multicolumn{3}{|c|}{ Vibración de tensión del doble enlace carbono-carbono } & - \\
\hline$v(\mathrm{COO}-)$ & & \multicolumn{3}{|c|}{$\begin{array}{l}\text { Vibración de tensión del enlace entre el carbono y } \\
\text { oxígenos del grupo carboxilo }\end{array}$} & - \\
\hline$\gamma(=\mathrm{CH})$ & & \multicolumn{3}{|c|}{$\begin{array}{l}\text { Deformación fuera del plano del enlace carbono- } \\
\text { hidrógeno de un alqueno }\end{array}$} & - \\
\hline$v(\mathrm{C}-\mathrm{H})$ & & \multicolumn{3}{|c|}{ Vibración de tensión del enlace carbono-hidrógeno } & - \\
\hline$-\mathrm{OCH}_{3}$ & & \multicolumn{3}{|c|}{ Grupo metoxi } & - \\
\hline$\Delta v(\mathrm{CO} 2-)$ & & \multicolumn{3}{|c|}{$\begin{array}{l}\text { Diferencia entre los valores de las vibraciones de tensión } \\
\text { asimétrica y simétrica de los enlaces del grupo carboxilo }\end{array}$} & - \\
\hline$v(\operatorname{Ln}-\mathrm{O})$ & & \multicolumn{3}{|c|}{ Vibración de tensión del enlace metal-oxígeno } & - \\
\hline$v_{\text {as }}$ & & \multicolumn{3}{|c|}{ Vibración de tensión asimétrica } & - \\
\hline$v_{\text {as }}$ & & \multicolumn{3}{|c|}{ Vibración de tensión simétrica } & - \\
\hline $\mathrm{Ce}_{2}\left(\mathrm{CO}_{3}\right)_{3}$ & & \multicolumn{3}{|c|}{ Carbonato de cerio } & - \\
\hline $\mathrm{Sm}_{2}\left(\mathrm{CO}_{3}\right)_{3}$ & & \multicolumn{3}{|c|}{ Carbonato de samario } & - \\
\hline $\mathrm{Nd}_{2}\left(\mathrm{CO}_{3}\right)_{3}$ & & \multicolumn{3}{|c|}{ Carbonato de neodimio } & - \\
\hline $\mathrm{Ce}_{2} \mathrm{O}_{3}$ & & \multicolumn{3}{|c|}{ Oxido de cerio } & - \\
\hline $\mathrm{Sm}_{2} \mathrm{O}_{3}$ & & \multicolumn{3}{|c|}{ Óxido de samario } & - \\
\hline $\mathrm{Nd}_{2} \mathrm{O}_{3}$ & & \multicolumn{3}{|c|}{ Óxido de neodimio } & - \\
\hline $\mathrm{Nd}_{2} \mathrm{O}_{2} \mathrm{CO}_{3}$ & & \multicolumn{3}{|c|}{ Carbonato óxido de neodimio } & - \\
\hline Unidades & \multicolumn{2}{|c|}{ Significado } & Unidades & \multicolumn{2}{|l|}{ Significado } \\
\hline h & \multicolumn{2}{|l|}{ Hora } & $\mu \mathrm{m}$ & \multicolumn{2}{|l|}{ Micrómetro } \\
\hline $\min$ & \multicolumn{2}{|l|}{ Minuto } & $\mathrm{mL}$ & Mililitro & \\
\hline eV & \multicolumn{2}{|c|}{ Electronvoltio } & $\mathrm{mmol}$ & Milimol & \\
\hline${ }^{\circ} \mathrm{C}$ & \multicolumn{2}{|c|}{ Grado celsius } & $\mathrm{Mg}$ & Miligramo & \\
\hline $\mathrm{mm}$ & \multicolumn{2}{|c|}{ Milímetro } & $\mathrm{cm}^{-1}$ & Centímetro a la menos & uno \\
\hline
\end{tabular}

\section{Anexo 2. Espectros FT-IR}

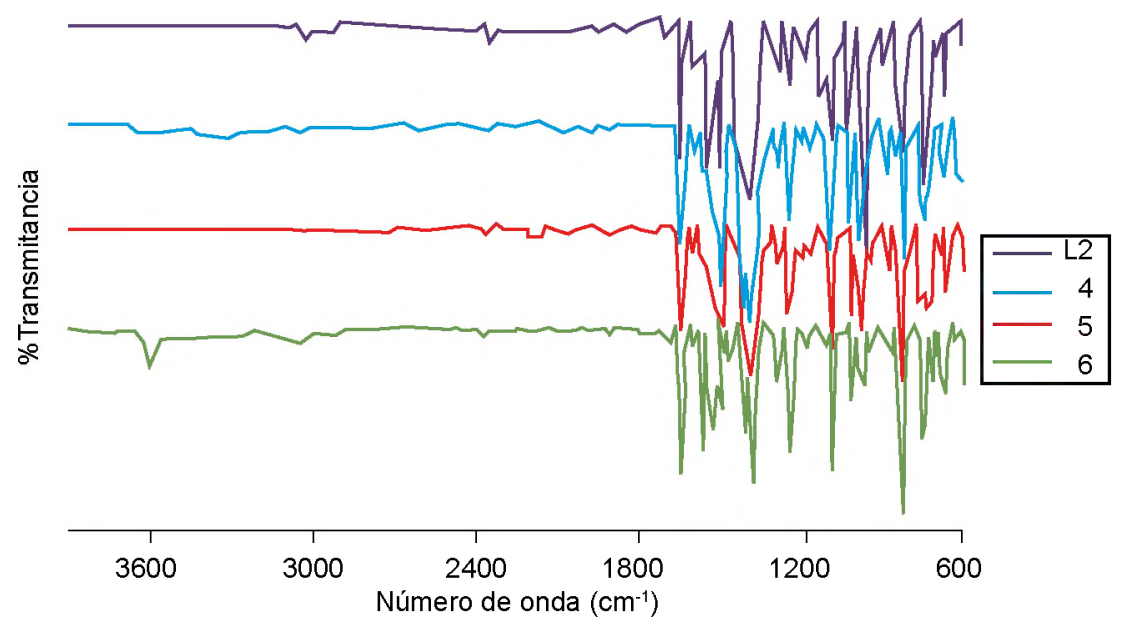

Figura 1. Comparación de los espectros FT-IR del ligando cinamato de sodio ( $L 2)$ y los complejos $\left[\mathrm{Ce}(\mathrm{L} 2)_{s} \cdot \mathrm{H}_{2} \mathrm{O}\right](4),\left[\mathrm{Nd}(\mathrm{L} 2)_{3}\right](5),\left[\mathrm{Sm}(\mathrm{L} 2)_{3}\right] 2 \mathrm{H}_{2} \mathrm{O}(6)$. 


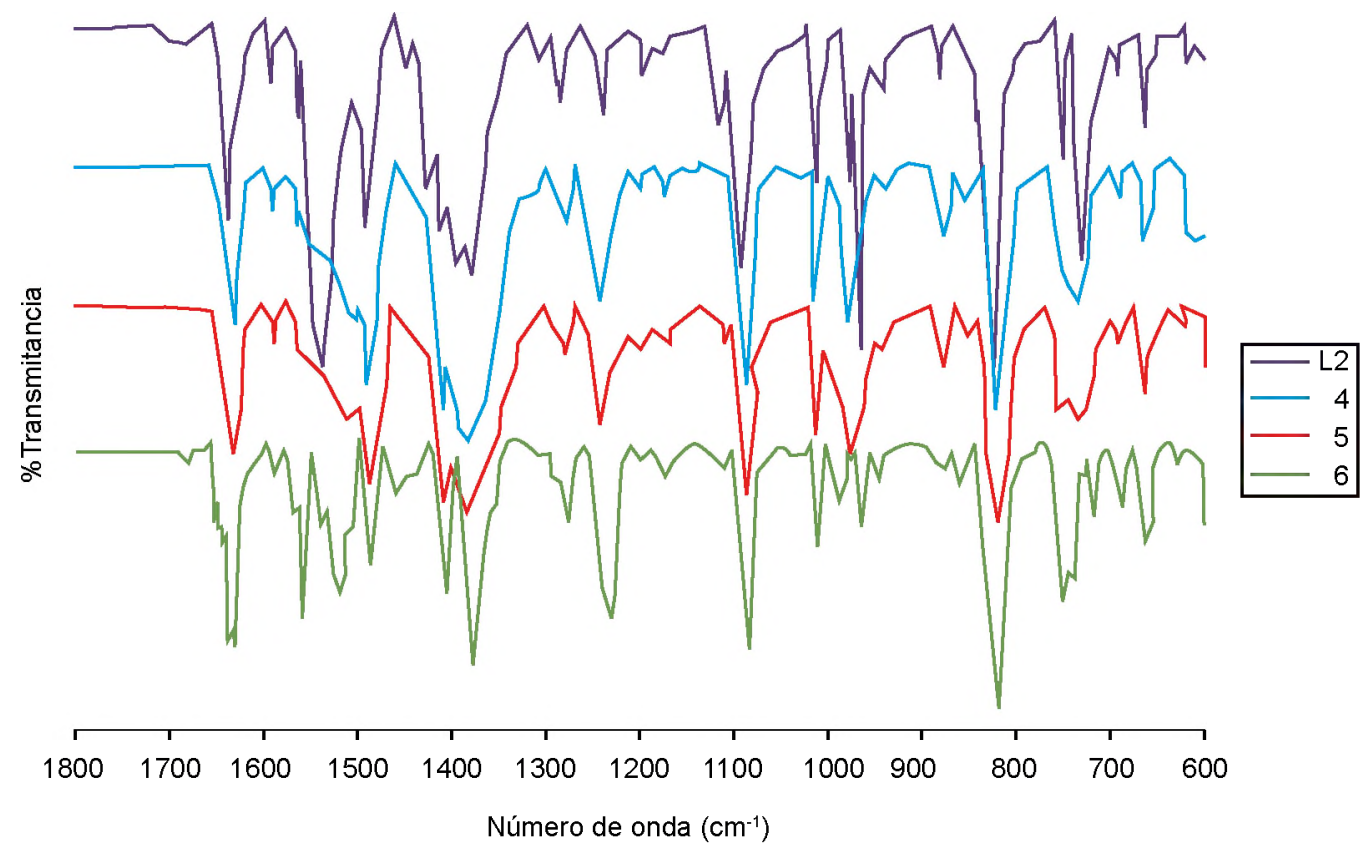

Figura 2. Expansión de la región dactilar de los espectros FT-IR del ligando cinamato de sodio (L2) y los complejos [Ce(L2)3 $\left.\mathrm{H}_{2} \mathrm{O}\right](4),[\mathrm{Nd}(\mathrm{L} 2) 3](5),[\mathrm{Sm}(\mathrm{L} 2) 3]_{2} \mathrm{H}_{2} \mathrm{O}(6)$.

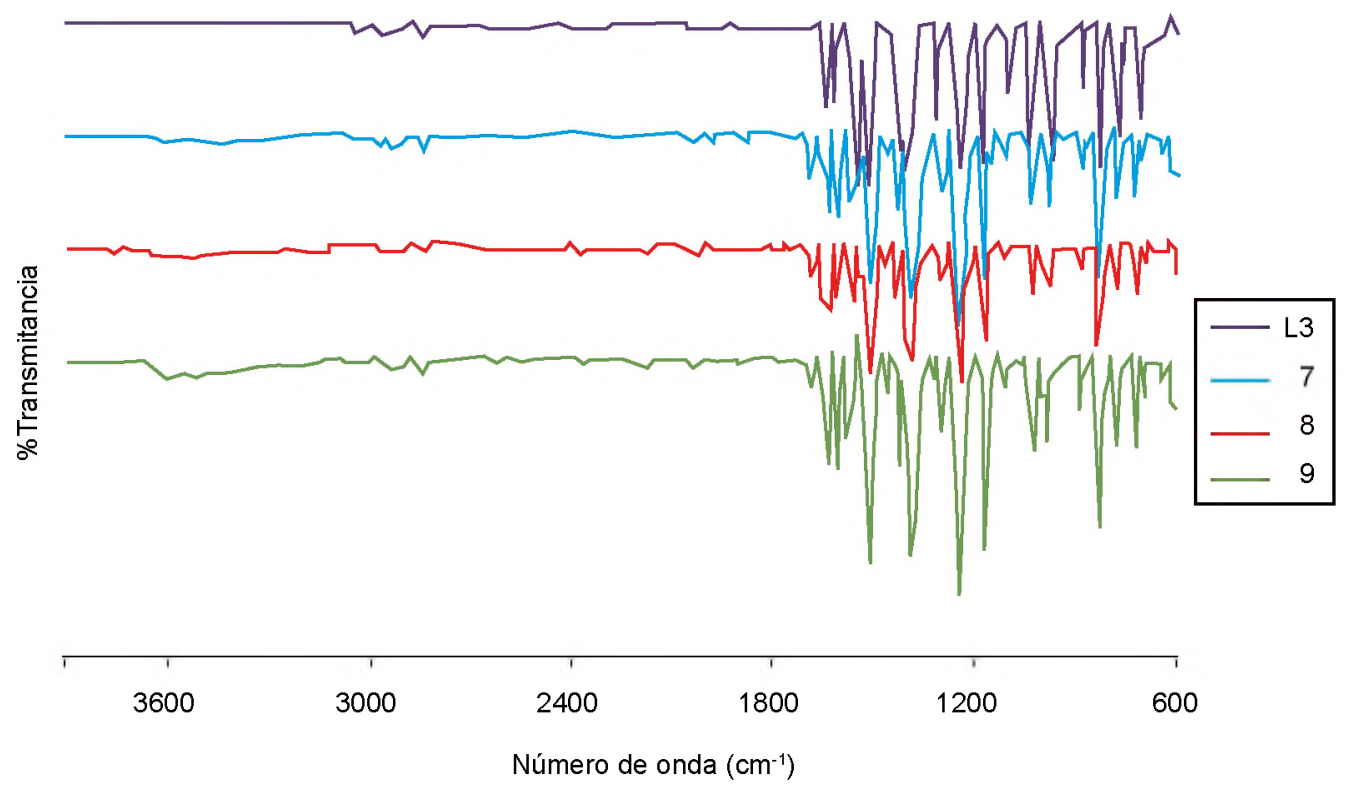

Figura 3. Comparación de los espectros FT-IR del ligando cinamato de sodio ( $L 3$ ) y los complejos $\left[\mathrm{Ce}(\mathrm{L3})_{3} \cdot \mathrm{H}_{2} \mathrm{O}\right] \cdot \mathrm{H}_{2} \mathrm{O}(7),\left[\mathrm{Nd}(\mathrm{L3})_{3}\right] \cdot \mathrm{H}_{2} \mathrm{O}(8),\left[\mathrm{Sm}(\mathrm{L} 3)_{3} 2 \mathrm{H}_{2} \mathrm{O}\right](9)$. 


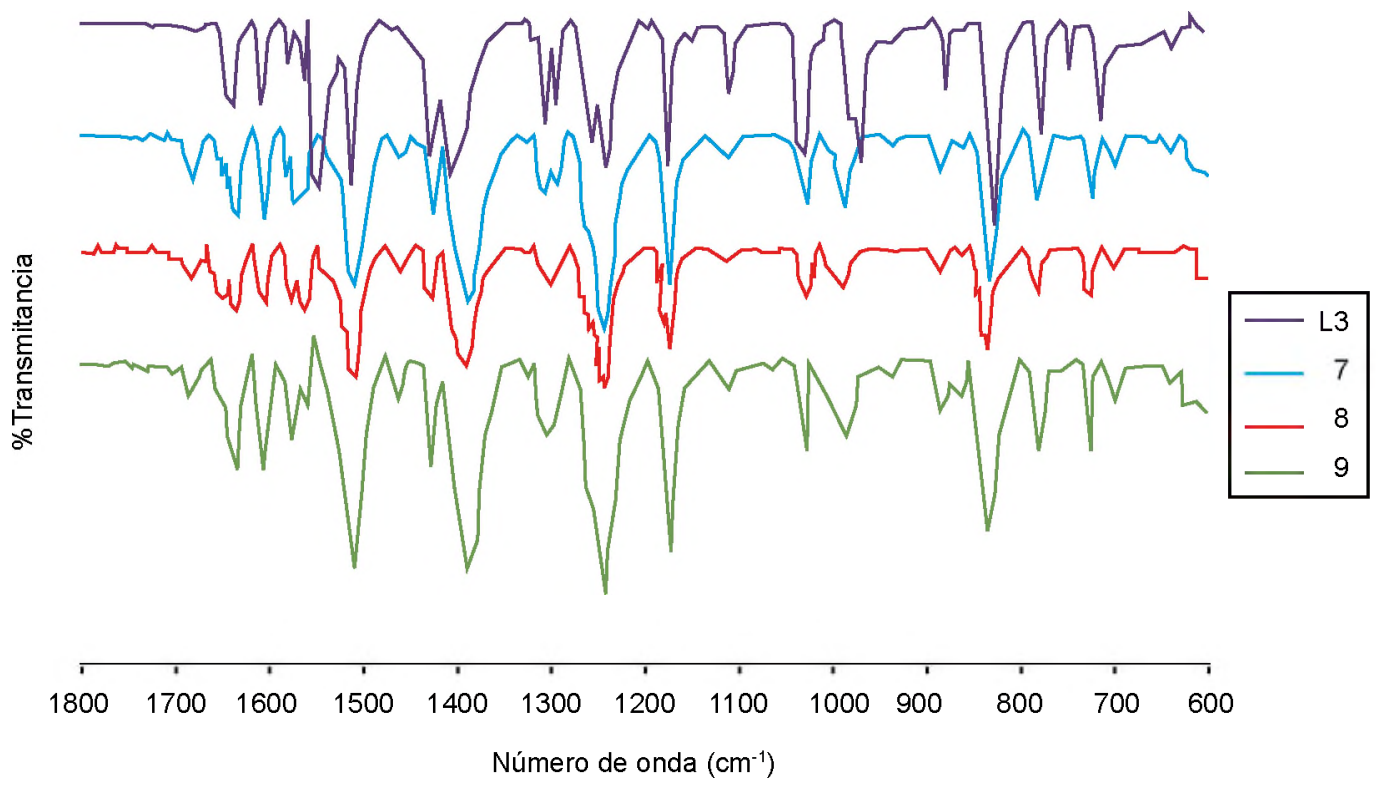

Figura 4. Expansión de la región dactilar de los espectros FT-IR del ligando cinamato de sodio (L3) y los complejos [Ce( $\left.\left(\mathrm{L}_{3}\right)_{3} \cdot \mathrm{H}_{2} \mathrm{O}\right] \cdot \mathrm{H}_{2} \mathrm{O}(7),\left[\mathrm{Nd}(\mathrm{L} 3)_{3}\right] \cdot \mathrm{H}_{2} \mathrm{O}(8),\left[\mathrm{Sm}(\mathrm{L} 3)_{3} 2 \mathrm{H}_{2} \mathrm{O}\right](9)$.

\section{Anexo 3. Análisis termogravimétricos}

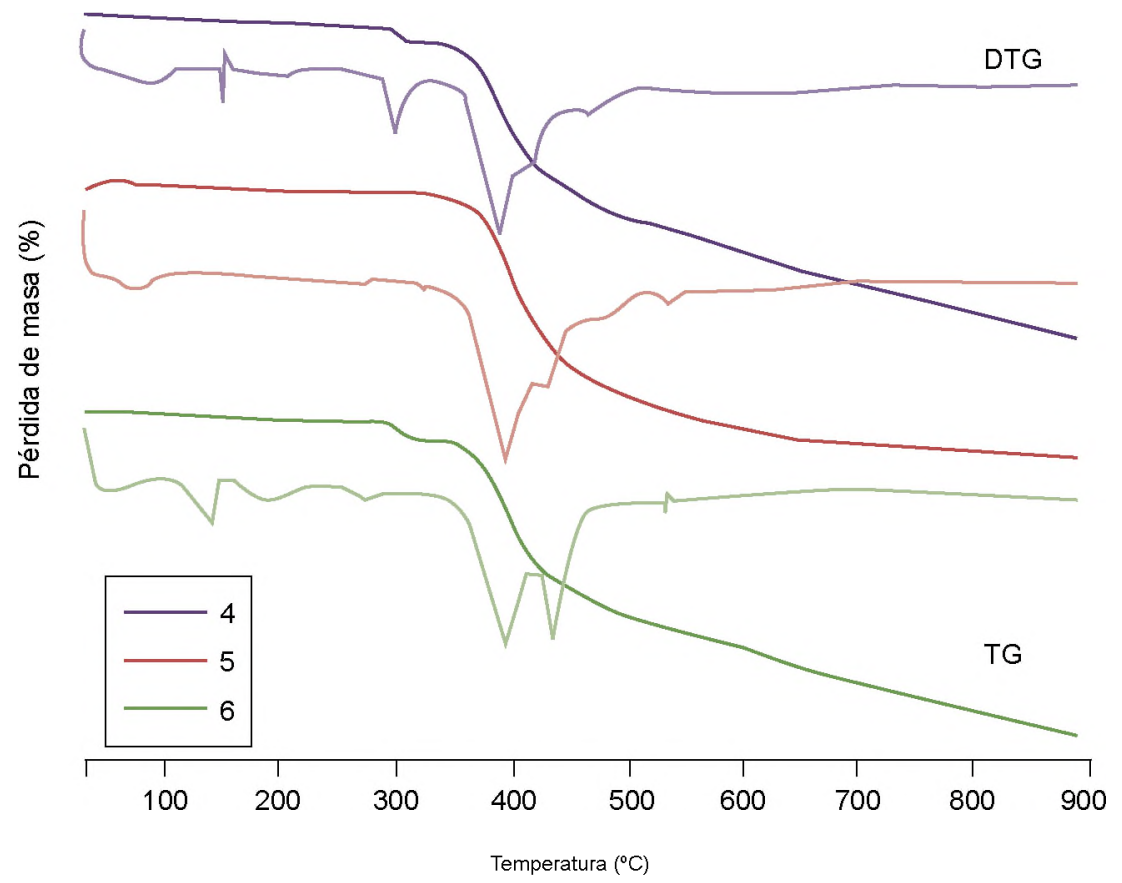

Figura 5. Curvas TG-DTG de los complejos [Ce(L2)3. $\left.\mathrm{H}_{2} \mathrm{O}\right](4)$, [Nd(L2)3] (5), [Sm(L2)3] $2 \mathrm{H}_{2} \mathrm{O}(6)$. 


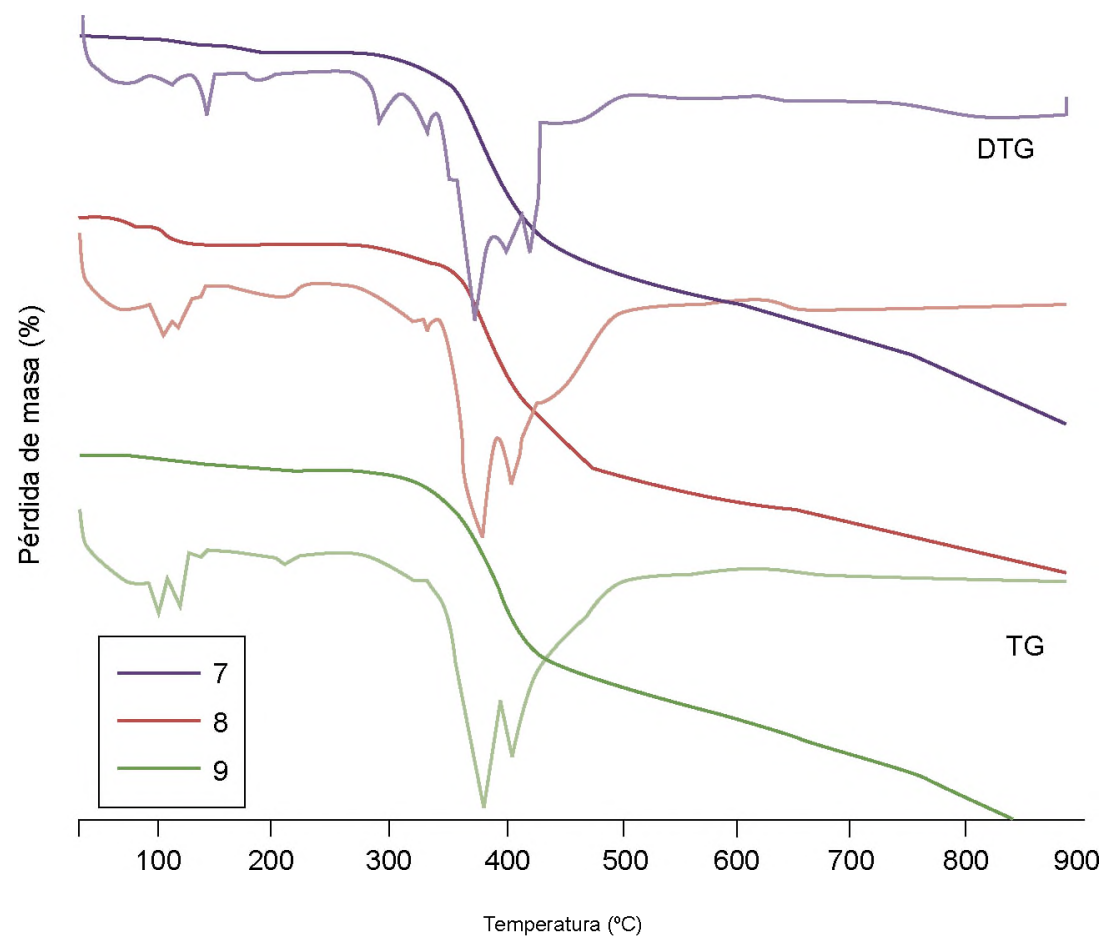

Figura 6. Curvas TG-DTG de los complejos $\left[\mathrm{Ce}(\mathrm{L} 3)_{3} \cdot \mathrm{H}_{2} \mathrm{O}\right] \cdot \mathrm{H}_{2} \mathrm{O}(7),\left[\mathrm{Nd}(\mathrm{L} 3)_{3}\right] \cdot \mathrm{H}_{2} \mathrm{O}(8)$, $\left[\mathrm{Sm}(\mathrm{L} 3)_{3}{ }_{2} \mathrm{H}_{2} \mathrm{O}\right](9)$.

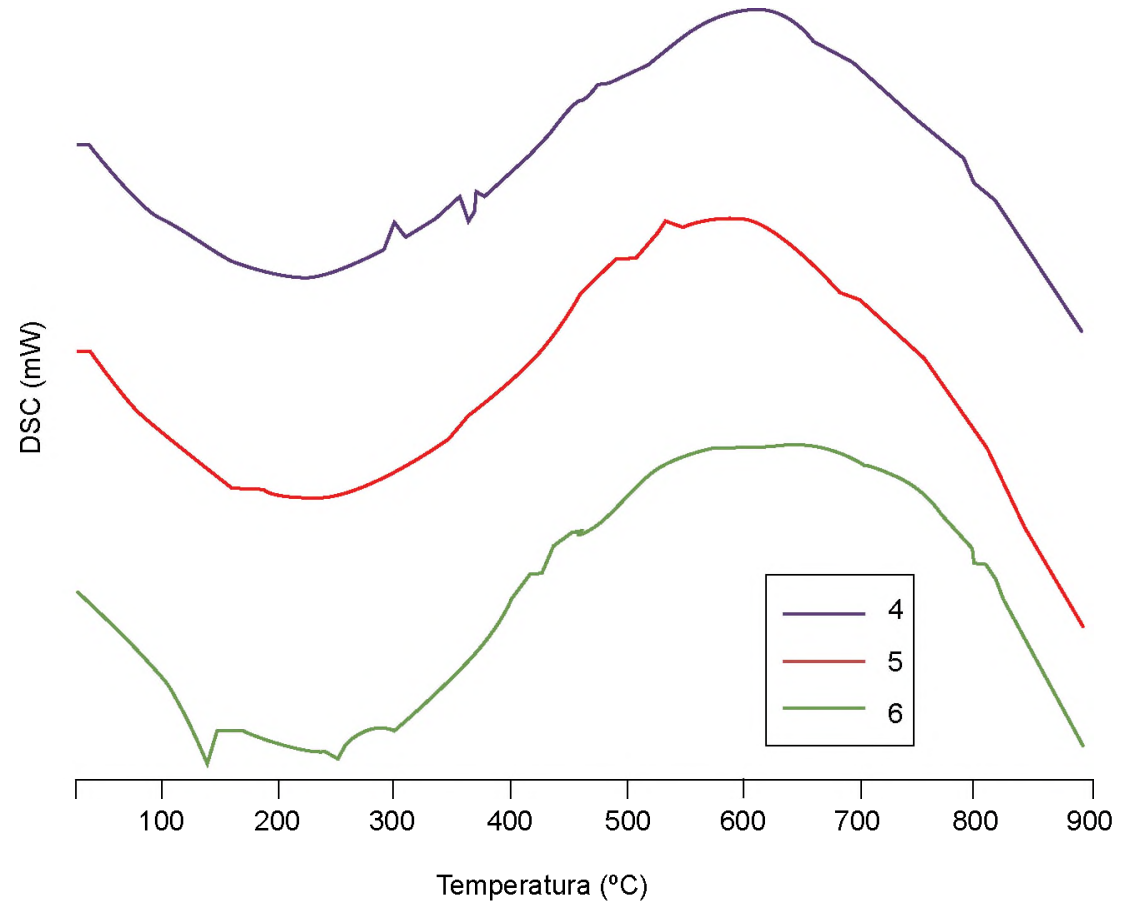

Figura 7. Curvas DSC de los complejos [Ce(L2)3 $\left.3 \mathrm{H}_{2} \mathrm{O}\right](4),[\mathrm{Nd}(\mathrm{L} 2) 3]$ (5), $\left[\mathrm{Sm}(\mathrm{L} 2)_{3}\right] 2 \mathrm{H}_{2} \mathrm{O}(6)$. 


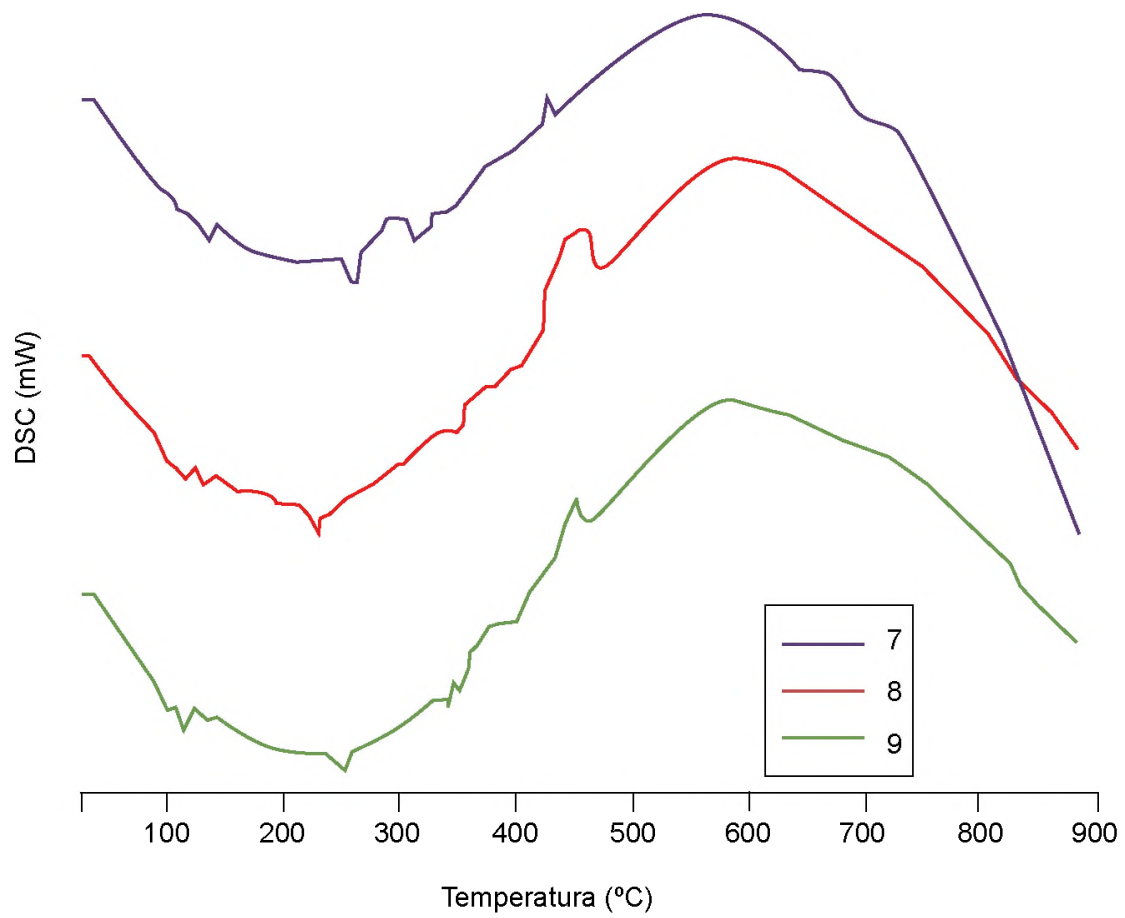

Figura 8. Curvas DSC de los complejos [Ce(L3)3 $\left.\mathrm{H}_{2} \mathrm{O}\right] \cdot \mathrm{H}_{2} \mathrm{O}(7),[\mathrm{Nd}(\mathrm{L} 3) 3] \cdot \mathrm{H}_{2} \mathrm{O}(8),\left[\mathrm{Sm}\left(\mathrm{L}_{3}\right)_{3} \mathrm{CH}_{2} \mathrm{O}\right](9)$.

\section{Anexo 4. Espectros de masas}

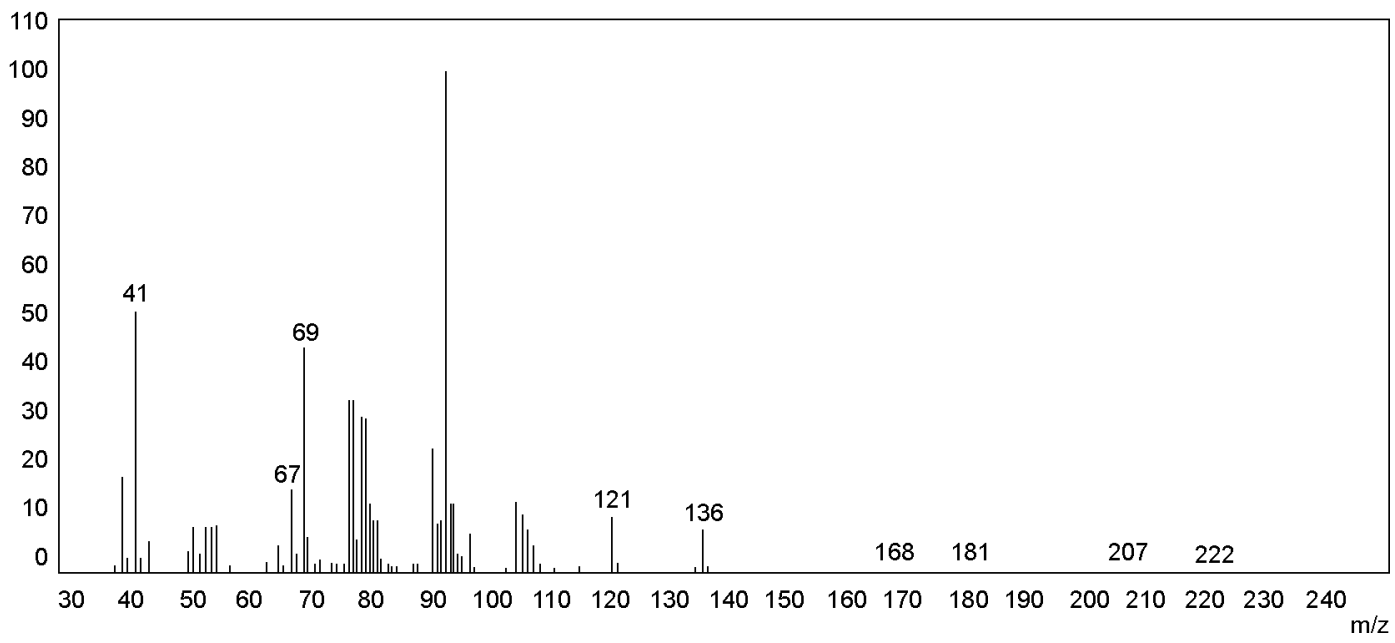

Figura 9. Espectro de masas del $\beta$-pineno obtenido experimentalmente. 


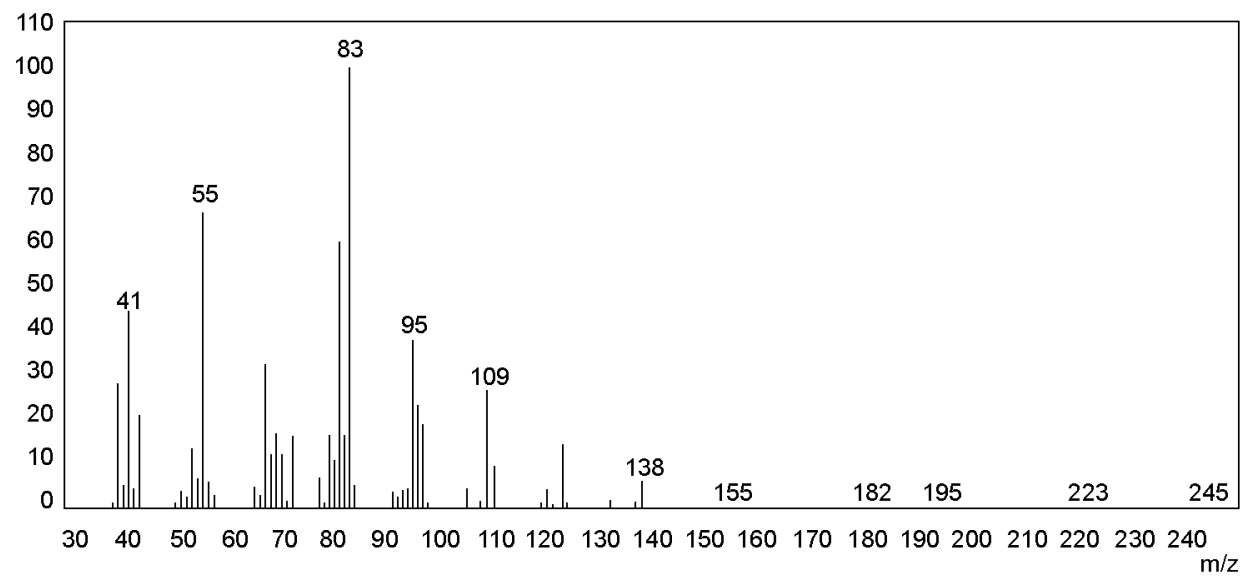

Figura 10. Espectro de masas de la nopinona obtenido experimentalmente.

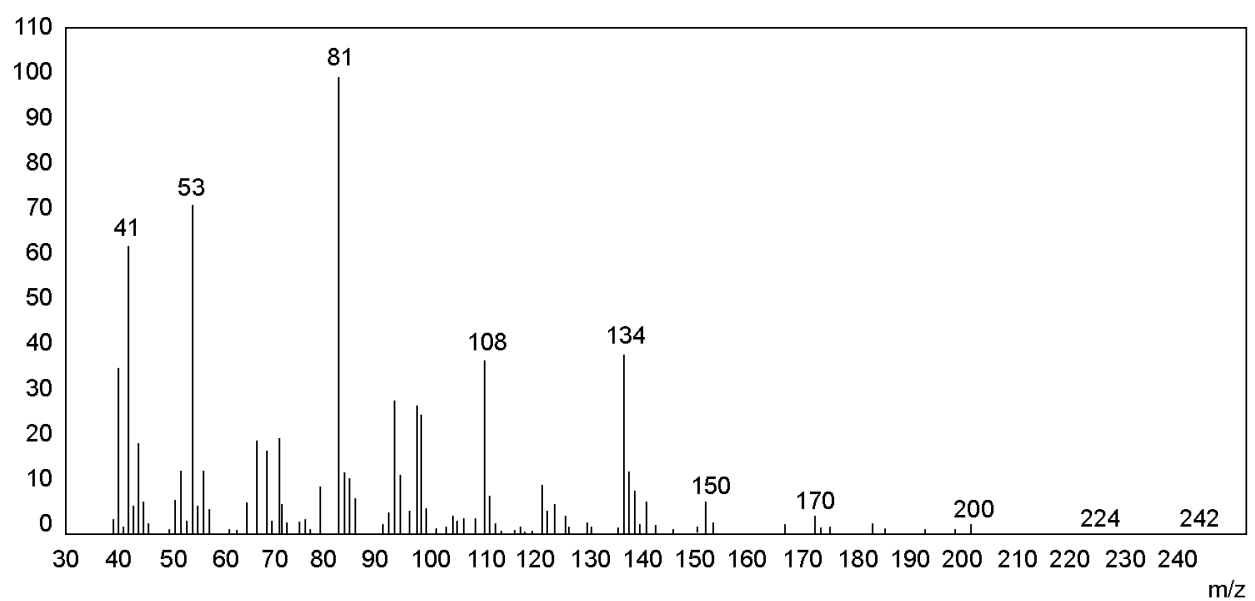

Figura 11. Espectro de masas de la pinocarvona obtenido experimentalmente.

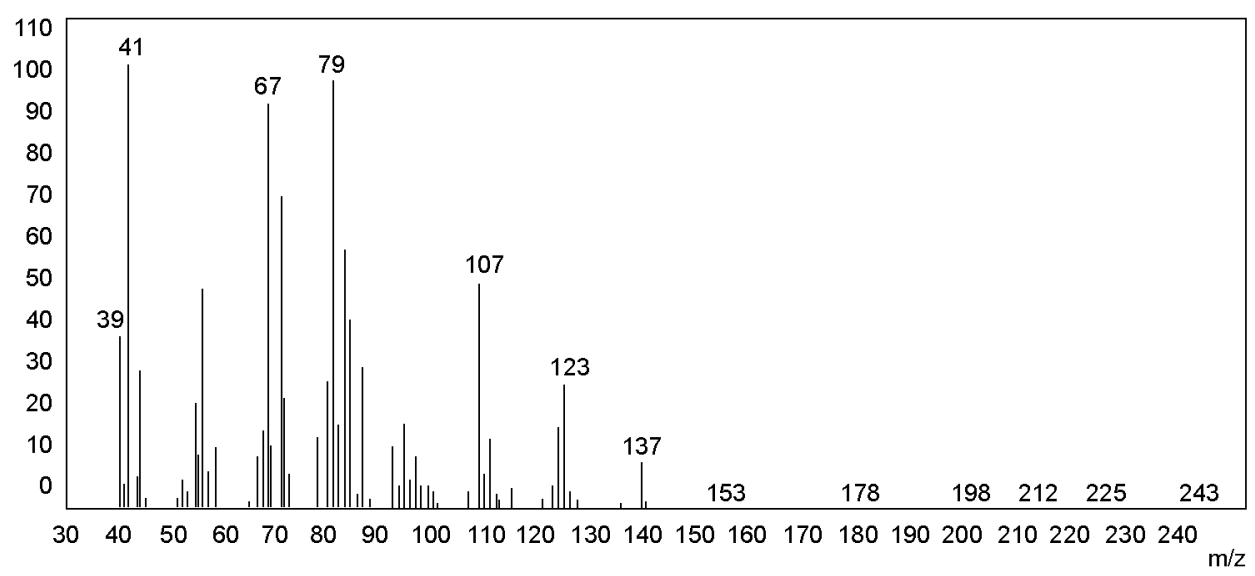

Figura 12. Espectro de masas del epoxi- $\beta$-pineno obtenido experimentalmente. 


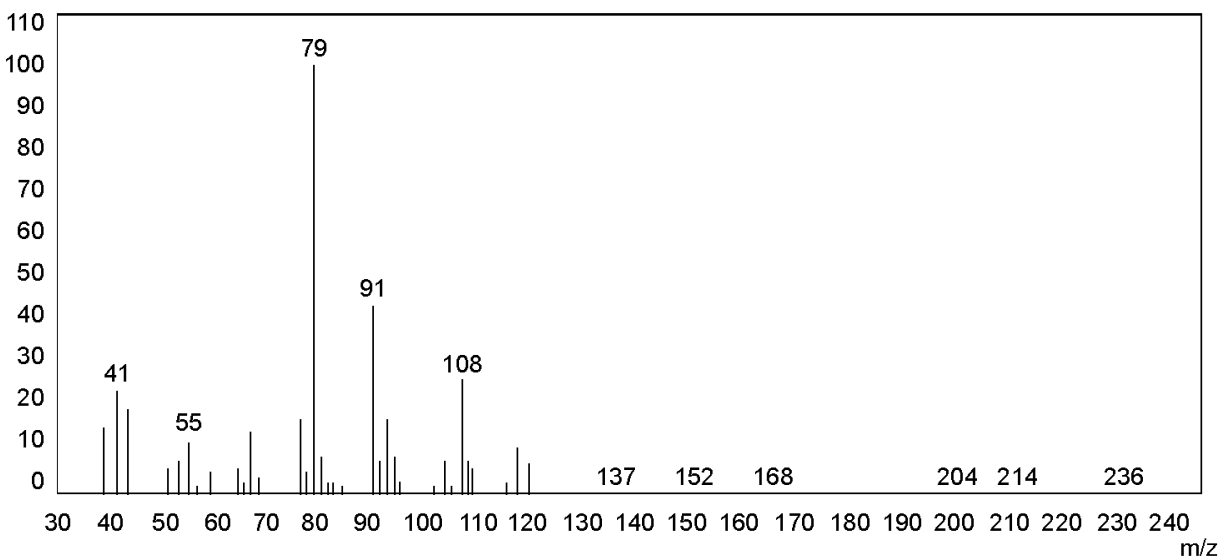

Figura 13. Espectro de masas del mirtenol obtenido experimentalmente.

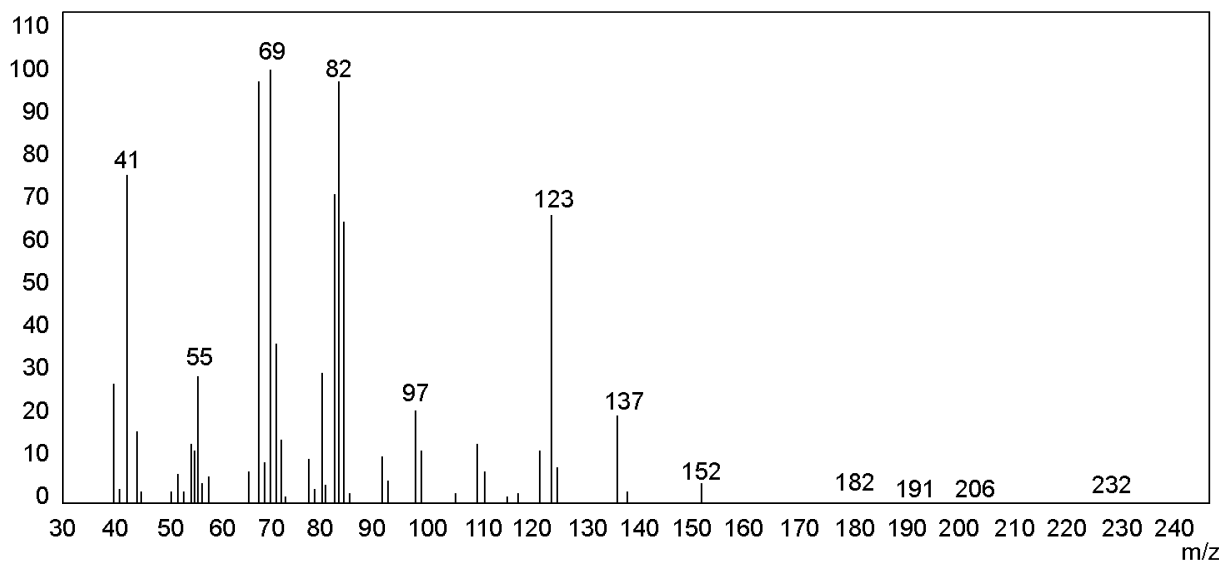

Figura 14. Espectro de masas del mirtenal obtenido experimentalmente.

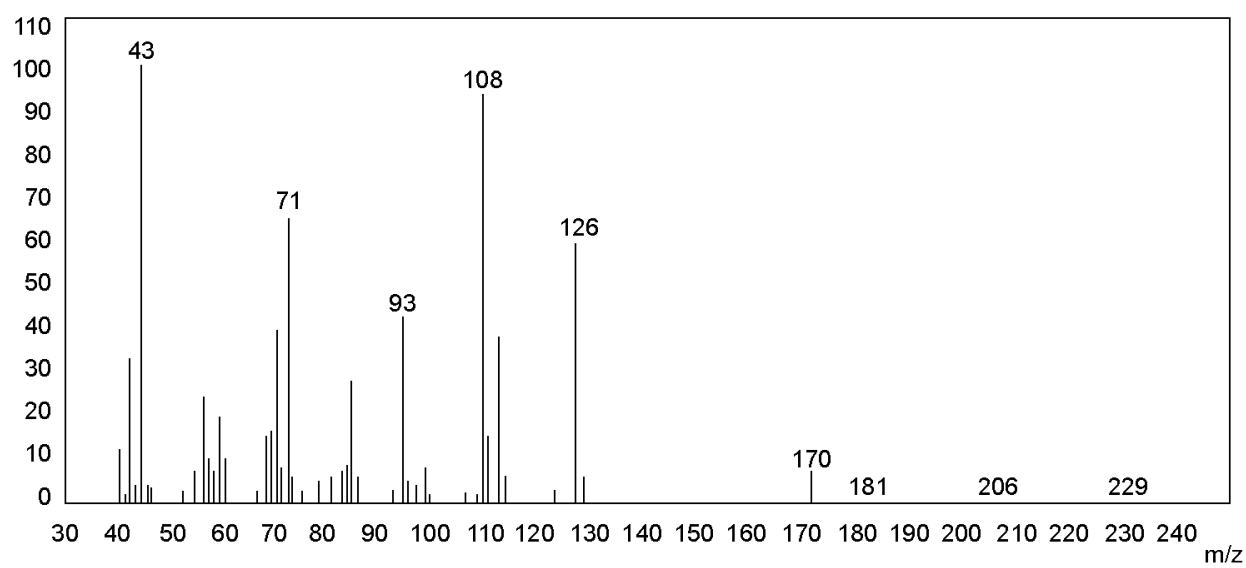

Figura 15. Espectro de masas del 2,3-pinanodiol obtenido experimentalmente. 


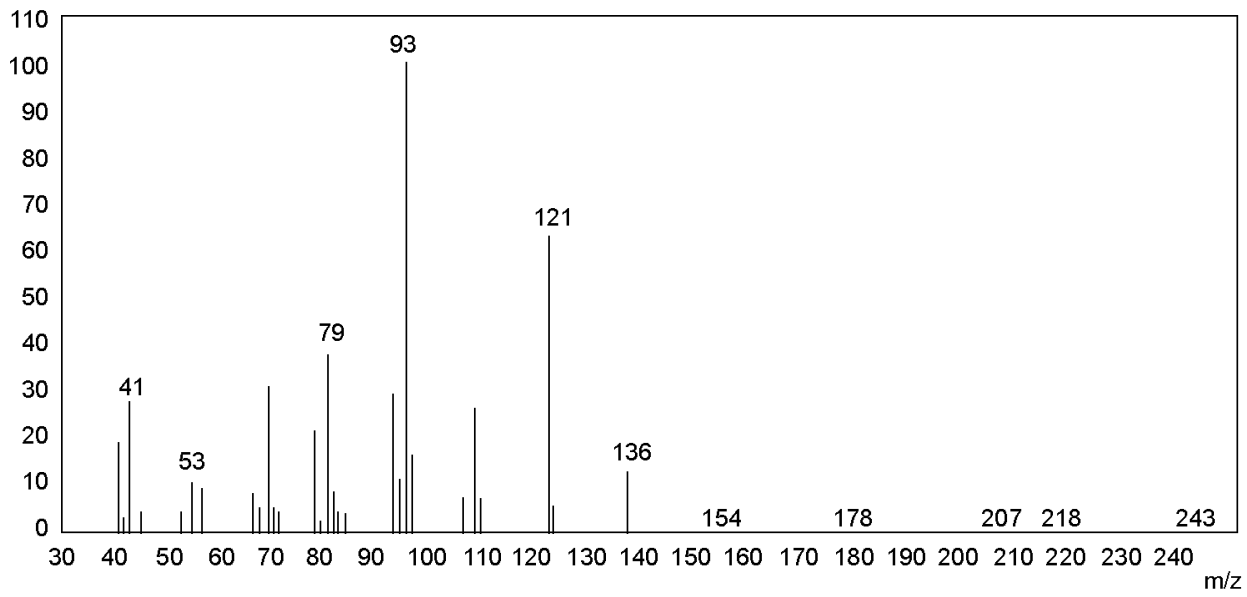

Figura 16. Espectro de masas del canfeno obtenido experimentalmente.

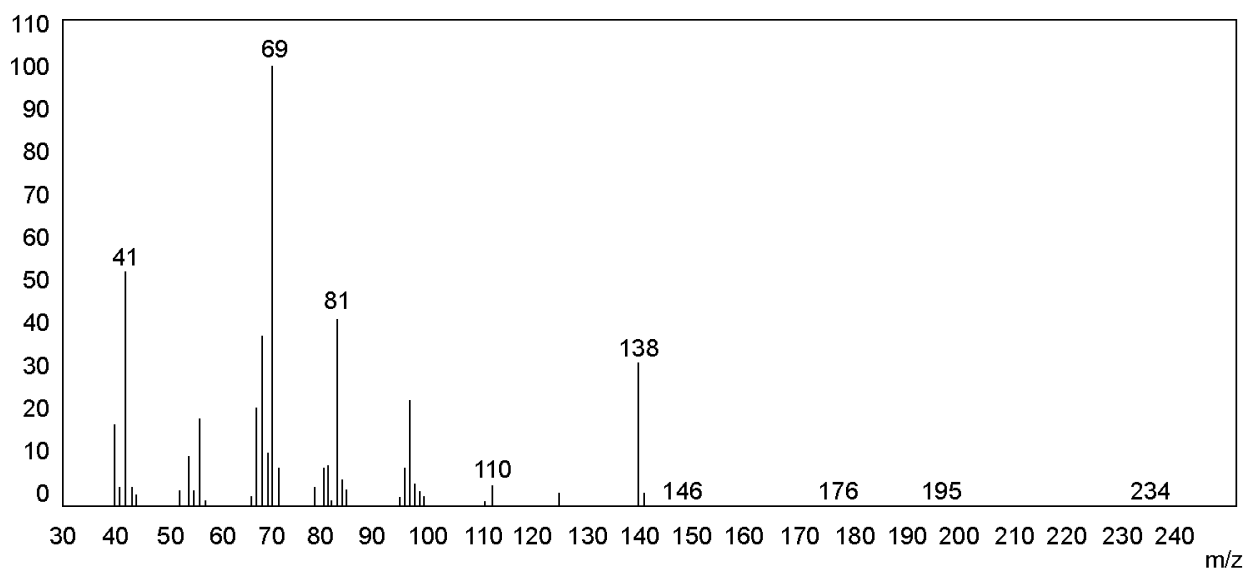

Figura 17. Espectro de masas de la 2-canfenilona obtenido experimentalmente.

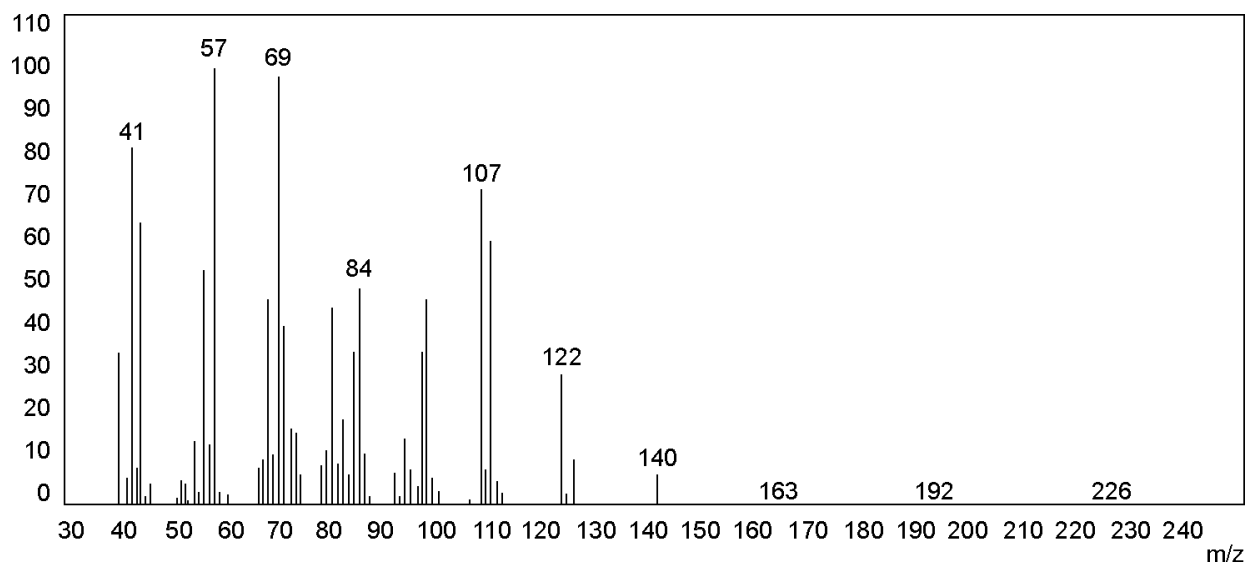

Figura 18. Espectro de masas del canfenilol obtenido experimentalmente. 


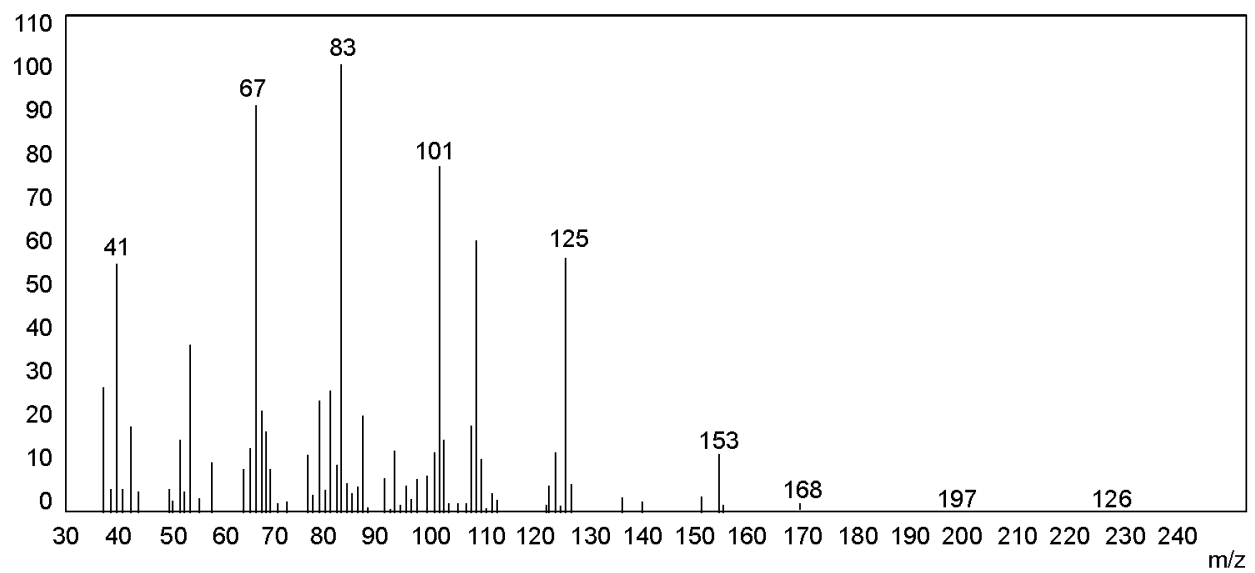

Figura 19. Espectro de masas del ácido 3-canfenilanocarboxílico obtenido experimentalmente.

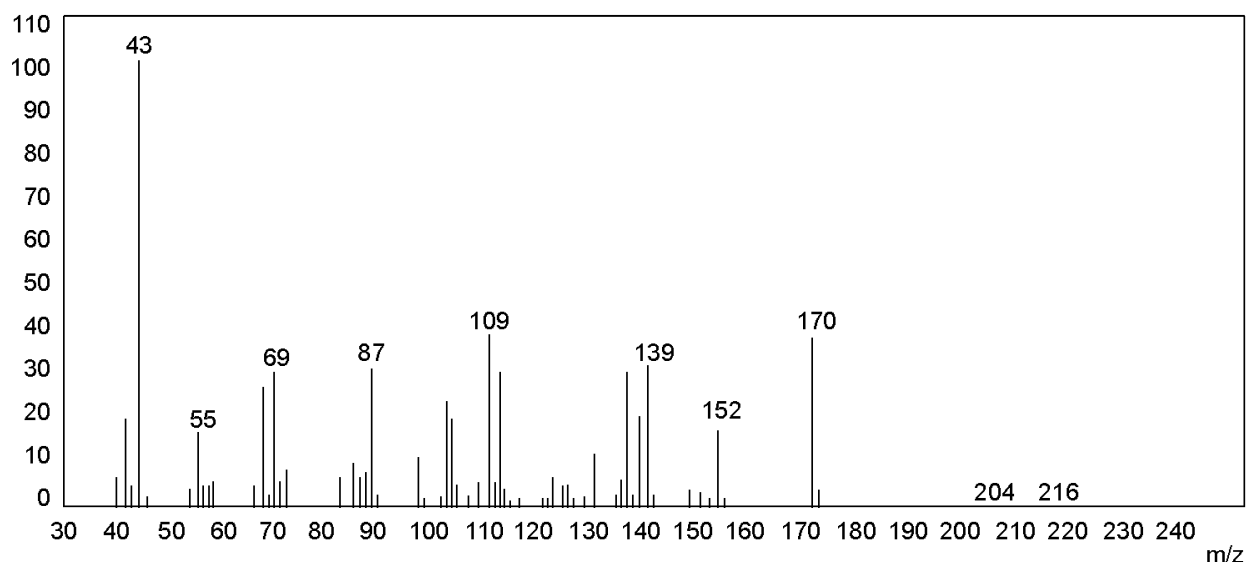

Figura 20. Espectro de masas del canfeno glicol obtenido experimentalmente. 